OPEN ACCESS

Edited by:

Hua Niu,

Affiliated Hospital of Guilin Medical

University, China

Reviewed by:

Xianyun Sun,

Institute of Microbiology, Chinese

Academy of Sciences, China

Sheng-hua Ying,

Zhejiang University, China

*Correspondence:

Jinkui Yang

jinkui960@ynu.edu.cn

${ }^{\dagger}$ These authors have contributed equally to this work

Specialty section:

This article was submitted to Microbes and Innate Immunity,

a section of the journal

Frontiers in Cellular and

Infection Microbiology

Received: 29 November 2021 Accepted: 29 December 2021 Published: 25 January 2022

Citation:

Zhou D, Zhu Y, Bai N, Xie M, Zhang K-Q and Yang J (2022) Aolatg1 and Aolatg13 Regulate Autophagy and Play Different Roles in Conidiation,

Trap Formation, and Pathogenicity

in the Nematode-Trapping Fungus

Arthrobotrys oligospora.

Front. Cell. Infect. Microbiol. 11:824407.

doi: 10.3389/fcimb.2021.824407

\section{Aolatg1 and Aolatg13 Regulate Autophagy and Play Different Roles in Conidiation, Trap Formation, and Pathogenicity in the Nematode-Trapping Fungus Arthrobotrys oligospora}

\author{
Duanxu Zhou ${ }^{1,2+}$, Yingmei Zhu ${ }^{1,2+}$, Na Bai ${ }^{1,2}$, Meihua Xie ${ }^{1}$, Ke-Qin Zhang ${ }^{1,2}$ and Jinkui Yang ${ }^{1,2 *}$ \\ 1 State Key Laboratory for Conservation and Utilization of Bio-Resources, and Key Laboratory for Microbial Resources of the \\ Ministry of Education, Yunnan University, Kunming, China, ${ }^{2}$ School of Life Sciences, Yunnan University, Kunming, China
}

Autophagy is a conserved cellular recycling and trafficking pathway in eukaryotes that plays an important role in cell growth, development, and pathogenicity. Atg1 and Atg13 form the Atg1-Atg13 complex, which is essential for autophagy in yeast. Here, we characterized the roles of the Aolatg1 and Aolatg13 genes encoding these autophagyrelated proteins in the nematode-trapping fungus Arthrobotrys oligospora. Investigation of the autophagy process by using the AoAtg8-GFP fusion protein showed that autophagosomes accumulated inside vacuoles in the wild-type (WT) A. oligospora strain, whereas in the two mutant strains with deletions of Aolatg1 or Aolatg13, GFP signals were observed outside vacuoles. Similar results were observed by using transmission electron microscopy. Furthermore, deletion of Aolatg1 caused severe defects in mycelial growth, conidiation, conidial germination, trap formation, and nematode predation. In addition, transcripts of several sporulation-related genes were significantly downregulated in the $\Delta$ Aolatg1 mutant. In contrast, except for the altered resistance to several chemical stressors, no obvious differences were observed in phenotypic traits between the WT and $\Delta$ Aolatg13 mutant strains. The gene ontology analysis of the transcription profiles of the WT and $\Delta$ Aolatg1 mutant strains showed that the set of differentially expressed genes was highly enriched in genes relevant to membrane and cellular components. The Kyoto Encyclopedia of Genes and Genomes analysis indicated that differentially expressed genes were highly enriched in those related to metabolic pathways, autophagy and autophagy-related processes, including ubiquitinmediated proteolysis and SNARE interaction in vesicular transport, which were enriched during trap formation. These results indicate that Aolatg1 and Aolatg13 play crucial roles in the autophagy process in A. oligospora. Aolatg1 is also involved in the regulation of asexual growth, trap formation, and pathogenicity. Our results highlight the importance of Aolatg1 in the growth and development of $A$. oligospora, and provide a basis for 
elucidating the role of autophagy in the trap formation and pathogenicity of nematodetrapping fungi.

Keywords: Arthrobotrys oligospora, autophagy-related gene (atg), conidiation, trap formation, nematode predation, transcriptomic analysis

\section{INTRODUCTION}

Autophagy is a conserved degradation pathway that controls the homeostasis of the cellular environment by degrading organelles and proteins (Kroemer and Levine, 2008). Autophagy is induced in response to nutrient starvation and mediated by the cytoplasm-tovacuole targeting (Cvt) pathway, which is responsible for specific sorting of proteins to vacuoles (Ying and Feng, 2019). Autophagy is tightly controlled by autophagy-related genes (atg), and approximately $42 \mathrm{Atg}$ proteins have been identified in Saccharomyces cerevisiae (Zhu et al., 2019; Ying and Feng, 2019). Based on their specific functions, Atg proteins have been classified into six different groups: the Atg1 kinase complex, the Atg18-Atg2 complex, the phosphatidylinositol 3-kinase complex, the Atg12 conjugation system, the Atg8 conjugation system, and the Atg9 recycling complex (Nanji et al., 2017; Nakatogawa, 2020). The Atg1 complex is the initiator kinase complex for autophagy that serves as a scaffold to recruit downstream factors and regulate their functions via phosphorylation of serine or threonine residues (Nakatogawa, 2020). Atg1 is a serine/threonine protein kinase and the only enzymatic subunit of the Atg1 complex. Atg1 activity is essential for the turnover and recycling of other Atg proteins after the formation of autophagosomes (Cheong et al., 2008). Atg13 functions as a major positive regulator of Atg1 protein kinase and is highly phosphorylated under nutrient-rich conditions by protein kinase A and the target of rapamycin complex 1 (TORC1) (Kamada et al., 2000; Stephan et al., 2009). Under conditions of nutrient starvation or the presence of the specific inhibitor rapamycin, Atg13 is dephosphorylated, which allows its interaction with Atg1 and Atg17, resulting in the formation of the Atg1 complex and activation of the Atg1 kinase via its autophosphorylation (Memisoglu et al., 2019; Nakatogawa, 2020). In S. cerevisiae, the Atg1 complex consists of the protein kinase Atg1, the TORC1 substrate Atg13, and the trimeric Atg17-Atg31-Atg29 scaffolding subcomplex, which triggers autophagy when Atg1 and Atg13 assemble with the trimeric scaffold (Stjepanovic et al., 2014). The Atg1 complex mediates autophagosome formation by initiating phagophore assembly and localizing the downstream phosphatidylinositol 3-kinase complex, Atg9, and the ubiquitinlike conjugation systems to this membrane compartment (Chew et al., 2015).

In filamentous fungi, autophagy appears to be involved in nutrient recycling during starvation, and it has been suggested to regulate normal developmental processes. Several methods have been developed to visualize autophagy, such as transmission electron microscopy (TEM), GFP-Atg8 fusion protein, and probes for acidic compartment (Pollack et al., 2009). At present, the functions of Atg1 have been described in several filamentous fungi. For example, blockade of autophagy in the
$\Delta$ Mgatg1 mutant of Magnaporthe oryzae (syn. Magnaporthe grisea) impaired its ability to penetrate and infect the host (Liu et al., 2007). In Aspergillus oryzae, conidiation and development of aerial hyphae were suppressed in the $\Delta$ Aoatg1 mutant, so AoAtg1 was deemed to be essential for nonselective autophagy and the Cvt pathway (Yanagisawa et al., 2013). Similarly, disruption of Bbatg1 impaired autophagy, conidial yield, conidial germination, and virulence in Beauveria bassiana (Ying et al., 2016). Deletion of Bcatg1 impaired autophagy and dramatically suppressed vegetative growth, conidiation, and sclerotium formation in the $\Delta B$ catgl mutant of Botrytis cinerea (Ren et al., 2017). Unlike Atg1, Atg13 has been characterized only in a limited number of fungi. In $M$. oryzae, the $\Delta$ Moatg13 mutant displayed the phenotype similar to that of the wild-type (WT) strain (Dong et al., 2009), whereas in A. oryzae, the number of conidia was lower in the $\Delta$ Aoatg13 mutant than in the WT strain (Kikuma and Kitamoto, 2011).

Nematode-trapping (NT) fungi are a specific filamentous group that can form unique mycelial structures (traps) for nematode predation, thus playing important roles in maintaining nematode population density in natural environments (Su et al., 2017). Arthrobotrys oligospora is a representative NT species that can live both saprophytically on organic matter and as a predator, by capturing tiny animals (Nordbring-Hertz et al., 2006). A. oligospora is used as the primary model for interactions between fungi and nematodes (Niu and Zhang, 2011). When stimulated by nematodes or other inducing factors, A. oligospora produces adhesive networks, indicating a switch from the saprophytic lifestyle to the predacious stage (Nordbring-Hertz, 2004; Yang et al., 2011). Since the sequencing of A. oligospora genome, an increasing number of studies has focused on the mechanism underlying trap formation, and several signaling proteins, such as regulators of G-protein, G-protein subunits and small GTPases, have been shown to regulate trap morphogenesis and lifestyle switch (Yang et al., 2018; Yang et al., 2020; Bai et al., 2021; Ma et al., 2021; Yang et al., 2021). Moreover, three orthologous Atg proteins have been identified in A. oligospora: deletion of Aolatg8 blocked autophagy and abolished conidiation and trap formation (Chen et al., 2013), whereas deletion of Aolatg4 and Aolatg5 impaired autophagy and resulted in a reduction in conidia yields, cell nucleus number, and trap production (Zhou et al., 2020; Zhou et al., 2021). Despite their conserved features, autophagy proteins have different functions in fungi that are highly divergent morphology and lifestyle (Pollack et al., 2009; Ying and Feng, 2019).

In this study, to further probe biological impact of autophagy on the growth, development, and differentiation of NT fungi, the Atg1 and Atg13 homologs, which govern the first step of autophagy, were characterized in A. oligospora by gene disruption, phenotypic comparison, and transcriptomic 
analysis. Our results showed that Atg1 (AolAtg1) plays a crucial role in autophagy and underpins multiple phenotypic traits, whereas Atg13 (AolAtg13) plays a conserved role in autophagy and has a limited impact on the growth and development in $A$. oligospora. In addition, we compared transcriptional profiles of the WT and $\Delta$ Aolatg1 mutant strains obtained using RNA-seq technology, which provided insights into the regulation of autophagy in A. oligospora and other NT fungi.

\section{MATERIALS AND METHODS}

\section{Strains and Media}

The fungus A. oligospora (ATCC24927) and corresponding mutants were stored in the Microbial Library of the Germplasm Bank of wild species from Southwest China (Kunming, China). Potato dextrose agar (PDA), tryptone glucose (TG), and corn-maizena yeast extract (CMY) were prepared as described previously (Zhou et al., 2020; Zhou et al., 2021) and used to analyze fungal phenotypic traits. The complete medium $(\mathrm{CM})$ and $\mathrm{MM}-\mathrm{N}\left(0.5 \mathrm{~g} \mathrm{~L}^{-1} \mathrm{KCl}, 0.5 \mathrm{~g} \mathrm{~L}^{-1}\right.$ $\mathrm{MgSO}_{4}, 1.5 \mathrm{~g} \mathrm{~L}^{-1} \mathrm{KH}_{2} \mathrm{PO}_{4}, 0.1 \%$ trace element, and $10 \mathrm{~g} \mathrm{~L}^{-1}$ glucose; $\mathrm{pH}$ 6.5) were used to compare colony growth and induce autophagy under nitrogen starvation (Talbot et al., 1993). Plasmids pRS426 and pCSN44 were maintained in the Escherichia coli strain DH5 $\alpha$ (Takara, Shiga, Japan). S. cerevisiae (FY834) was used to screen the correctly recombined construct, and the selectionwas performed on the SC-Ura medium (Park et al., 2011). Caenorhabditis elegans (strain N2) was incubated on the oatmeal medium at $26^{\circ} \mathrm{C}$ for trap induction and bioassays.

\section{Sequence Analysis of AolAtg1 and AolAtg13 Proteins}

The homologous sequences of AolAtg1 (AOL_s00076g234) and AolAtg13 (AOL_s00215g74) were retrieved from the A. oligospora genome (Yang et al., 2011) using the sequences of the orthologous proteins Atg1 (NP_011335) and Atg13 (NP_015511) in $S$. cerevisiae. The molecular mass and isoelectric point of the proteins were calculated using the online tool Compute $\mathrm{pI} / \mathrm{Mw}$ (https://web.expasy.org/compute_pi/), and the conserved protein domains were predicted using InterPro (http://www.ebi.ac.uk/ interpro/). The orthologs of AolAtg1 and AolAtg13 from other fungi were examined by BlastP, and the similarity between Atg1 or Atg13 homologs was analyzed using DNAman software (Version 6). A neighbor-joining tree was constructed using Mega software (7.0) (Kumar et al., 2016).

\section{Deletion of Aolatg1 and Aolatg13 Genes}

The atg genes of $A$. oligospora were deleted using the homologous recombination method (Tunlid et al., 1999; Park et al., 2011). The upstream and downstream sequences corresponding to the genes Aolatg1 and Aolatg13 in A. oligospora were amplified using paired primers (Supplementary Table S1). Subsequently, the $h p h$ cassette for hygromycin resistance was amplified using primers Hph-f and Hph-r (Supplementary Table S1). Then, three PCR fragments and a linearized pRS426 vector were co-transformed into the yeast strain FY834 via electroporation. The complete fragment for gene disruption was amplified from the recombinant plasmid pRS426-Atg-hph using primers AolAtg1-5f/AolAtg1-3r or AolAtg13-5f/AolAtg13-3r (Supplementary Table S1), and it was transformed into A. oligospora using the protoplast transformation method as described previously (Tunlid et al., 1999; Liu et al., 2021; Long et al., 2021). The putative transformants were selected on the PDAS medium containing $200 \mathrm{~g} \mathrm{~L}^{-1}$ hygromycin B (Amresco, Solon, United States) (Liu et al., 2020; Xie et al., 2021). The successful deletions of the Aolatg1 and Aolatg13 genes were confirmed using PCR amplification and Southern blotting analyses, as described previously (Xie et al., 2019; Xie et al., 2020).

\section{Generation of the AoAtg8-GFP Fusion Protein}

The pPK2-GFP-Sur ( $\mathrm{pPK} 2$ ) vector harboring the green fluorescent protein (GFP) gene and the sulfonylurea resistance gene (sur) was used as a basic framework. The promoter fragment was amplified with primers AoP-f/AoP-r and inserted into the BsrGI/SpeI sites of the pPK2 vector, and the cDNA fragment of Aoatg8 (AOL_s00007g534) was amplified using the primer pair Atg8-f/Atg8-r (Supplementary Table S1) and then inserted into the BsrGI/SpeI sites of the pPK2 vector. The resultant $\mathrm{pPK} 2-\mathrm{GFP}-\mathrm{AoAtg} 8$ vector was inserted into the WT, $\Delta$ Aolatg1, and $\Delta$ Aolatg13 mutant strains using the protoplast transformation method (Tunlid et al., 1999). The putative transformants were cultured on plates supplemented with $10 \mu \mathrm{g} \mathrm{mL} \mathrm{m}^{-1}$ chlorimuron ethyl, and GFP signals were examined under a confocal laser scanning microscope.

\section{Comparison of Mycelial Growth and Stress Resistance}

The WT and mutant strains were incubated on PDA plates at $28^{\circ}$ $\mathrm{C}$ for 5 days, then transferred onto PDA, CMY, TG, CM, and MM-N plates to evaluate their growth rate under different nutritional conditions, and the diameters of colonies were determined at $24 \mathrm{~h}$ intervals (Zhou et al., 2020; Zhou et al., 2021). To determine the levels of stress resistance, the fungal strains were incubated on TG plates supplemented with or without (control) different concentrations of chemical stressors, including oxidative agents $\left(\mathrm{H}_{2} \mathrm{O}_{2}\right.$ and menadione) and osmotic agents $\left(\mathrm{NaCl}\right.$ and sorbitol) at $28^{\circ} \mathrm{C}$ for 7 days. Relative growth inhibition (RGI) values of the fungal strains were calculated as previously described (Zhen et al., 2018). To compare the lipid droplets (LDs) of fungal mycelia, the WT and mutant strains were incubated on PDA plates for 5 days and then stained with $10 \mu \mathrm{g} \mathrm{mL} \mathrm{mL}^{-1}$ BODIPY staining solution for $10 \mathrm{~min}$. LDs were observed using a fluorescence microscope.

\section{Comparison of Conidiation and Transcription of Sporulation- Related Genes}

To determine the spore yield, the WT and mutant strains were incubated on CMY plates at $28^{\circ} \mathrm{C}$ for 7 days, and then $5 \mathrm{~mL}$ of sterile water was added to each plate to harvest spores. Conidia were counted in $50 \mu \mathrm{L}$ aliquots using a hemocytometer. To 
analyze spore germination, $50 \mu \mathrm{L}$ suspensions $\left(10^{6}\right.$ spores per $\mathrm{mL}$ ) of WT and mutant strains were added to the MM-N liquid medium at $28^{\circ} \mathrm{C}$, and the number of germinated conidia was determined at 4, 8, and $12 \mathrm{~h}$ (Zhen et al., 2018).

To determine transcriptional levels of the sporulation-related genes, $50 \mu \mathrm{L}$ conidial suspension aliquots $\left(10^{6}\right.$ spores per $\left.\mathrm{mL}\right)$ of fungal strains were spread on CMY plates at $28^{\circ} \mathrm{C}$. The fungal samples were harvested from the cultures grown for 3, 5, and 7 days and stored at $-80^{\circ} \mathrm{C}$ for subsequent quantitative real-time PCR (qRT-PCR) analysis. The primers (Supplementary Table S2) for the target genes were designed using online software Primer3 (v0.4.0, https://bioinfo.ut.ee/primer3-0.4.0/). The expression of the A. oligospora $\beta$-tubulin gene (AOL_s00076g640) was used as the reference, and qRT-PCR analysis was performed as previously described (Yang et al., 2013). The transcript levels of each gene were analyzed using the $2^{-\Delta \Delta C t}$ method (Livak and Schmittgen, 2001).

\section{Confocal Microscopy and TEM Assays}

Hyphae of the WT and mutant strains were incubated in the CM medium at $28^{\circ} \mathrm{C}$ with gentle shaking at $180 \mathrm{r} \mathrm{min}^{-1}$ for 2 days, then transferred into the MM-N medium (nitrogen starvation) and incubated for $6 \mathrm{~h}$. Hyphae were collected to observe autophagosome formation using TEM and confocal microscopy (Lv et al., 2017). The lipophilic styryl dye FM4-64 (Invitrogen, Carlsbad, CA, USA) was used for vacuole staining of hyphal cells, as described previously (Ma et al., 2020).

\section{Trap Induction and Bioassay}

To induce trap formation, the conidia of fungal strains were collected from 7-day-old cultures on CMY plates, and $50 \mu \mathrm{L}$ suspensions $\left(10^{6}\right.$ spores per $\left.\mathrm{mL}\right)$ were incubated on water agar plates at $28^{\circ} \mathrm{C}$ for 3 days. Then, $\sim 300$ nematodes were added to each plate to induce trap formation, followed by microscopic observation of trap formation and nematode predation at $12 \mathrm{~h}$ intervals (Zhou et al., 2020).

\section{Transcriptomic Profile Analysis}

To probe the mechanism by which AolAtg1 regulates autophagy, the WT and $\triangle$ Aolatg1 mutant strains were incubated in the CMY medium at $28^{\circ} \mathrm{C}$, and the spores were harvested 7 days post incubation. Next, $1 \times 10^{5}$ spores were incubated on water agar plates at $28^{\circ} \mathrm{C}$ for $48 \mathrm{~h}$, and the hyphae were harvested. Two treatment groups with three independent biological replicates were collected at $0 \mathrm{~h}$ without nematodes and following $24 \mathrm{~h}$ incubation after the addition of 300-400 nematodes. The hyphae were sent to the Shanghai Meiji Biological Company (Shanghai, China) for RNA sequencing and data analysis.

High-quality RNA samples were used to construct a sequencing library that was sequenced on an Illumina HiSeq 4000 system (Illumina, San Diego, CA, USA). The data were analyzed using the Majorbio Cloud Platform (www.majorbio. com). To identify differentially expressed genes (DEGs), transcripts per kilobase million (TPM) values were calculated for each gene and compared between the WT and $\Delta$ Aolatg1 mutant strains. Gene abundance following RNA-seq was quantified by the expectation-maximization algorithm (Li and Dewey, 2011). Based on the quantitative expression results, DEGs were identified based on the following thresholds: $\mid \log 2$ ratio $\mid \geq 1$ and adjusted $P<0.05$. Gene Ontology (GO) and Kyoto Encyclopedia of Genes and Genomes (KEGG) analyses were performed to determine enrichment in GO terms of function classes and metabolic pathways in DEGs in comparison to the whole-transcriptome background. Sequence data were deposited in the National Center for Biotechnology Information (http://www.ncbi.nlm.nih.gov/) under the accession number PRJNA784322.

\section{Statistical Analysis}

All experimental data are presented as the mean \pm standard deviation (SD) of three biological replicates. Group effects were assessed by one-way analysis of variance followed by the Tukey's honestly significant difference (HSD) test. Prism 5 (GraphPad, San Diego, CA, USA) was used to generate plots and perform statistical analyses. Effects were considered statistically significant if $P<0.05$.

\section{RESULTS}

\section{Analysis of AolAtg1 and AolAtg13 Protein Sequences}

The sequences of the Atg1 and Atg13 homologs were retrieved from the A. oligospora genome. AolAtg1 was found to consist of 949 amino acids with a predicted molecular mass and isoelectric point of $103.6 \mathrm{kD}$ and 8.83 , respectively. AolAtg13 was shown to comprise 984 amino acids with a predicted molecular mass and isoelectric point of $105.8 \mathrm{kD}$ and 9.43, respectively. AolAtg1 contains a protein kinase domain (IPR000719) at the N-terminal and a serine/ threonine-protein kinase at the C-terminal (IPR022708). AolAtg13 contains the autophagy-related protein $13 \mathrm{~N}$-terminal domain (IPR018731). AolAtg1 shares a highly conserved protein sequence with homologous proteins from various NT fungi, having 94.2\% and 86.8\% identity to orthologous Atg1 from Arthrobotrys flagrans (syn. Duddingtonia flagrans) and Dactylellina haptotyla, respectively. AolAtg1 has moderate similarity (42.4-48.6\%) to orthologs from different filamentous fungi and $32.4 \%$ identity with Atg1 of S. cerevisiae (Supplementary Table S3). Relative to AolAtg1, AolAtg13 also has a high degree of identity (72.7-91.2\%) to orthologs from NT fungi, whereas its identity to orthologs from other filamentous fungi is low (23.2-29.5\%), and it has only $10.6 \%$ identity with Atg13 from S. cerevisiae (Supplementary Table S3). Phylogenetic analysis showed that orthologous Atg1 and Atg13 from filamentous fungi were divided into two clades, whereas orthologous Atg1 or Atg 13 from NT fungi were clustered together (Supplementary Figure S1).

\section{Aolatg1 and Aolatg13 Are Involved in Mycelial Growth and Lipid Metabolism}

Two independent positive transformants for Aolatg1 and Aolatg13 were screened and confirmed (Supplementary Figure S2), and their growth in various media was observed. The $\Delta$ Aolatg1 mutant displayed lower hyphal growth on CMY, TG, and PDA plates. The colony diameter of the WT strain was $7.75 \pm 0.25 \mathrm{~cm}$ on CMY plates at day 6 , whereas those of the 
$\Delta$ Aolatg1 and $\Delta$ Aolatg13 mutants were $6.61 \pm 0.26 \mathrm{~cm}$ and $6.94 \pm$ $0.30 \mathrm{~cm}$, respectively. Similarly, hyphal growth of the $\Delta$ Aolatg1 mutant was lower than that of the WT strain in the CM and MM-N plates (Supplementary Figure S3; Figures 1A, B). After staining with BODIPY staining solution, the LDs in hyphal cells were visualized. The hyphal cells of the WT strain contained numerous LDs, whereas in $\Delta$ Aolatg1 and $\Delta$ Aolatg13 mutants displayed remarkably fewer LDs (Figure 1C).

\section{Aolatg1 Regulates Sporulation and Spore Germination}

Aolatg1 deletion resulted in defective growth of aerial hyphae on CMY plates (Figure 2A), and the conidiophores of $\Delta$ Aolatg1 mutants became sparse compared to their number in the WT strain (Figure 2B). Thus, the loss of Aolatg1 caused a significant reduction in spore yield: the $\Delta$ Aolatg 1 mutant produced $1.16 \times$ $10^{6}$ conidia $\mathrm{cm}^{-2}$, which was $87.6 \%$ lower compared to the yield in the WT strain $\left(9.40 \times 10^{6}\right.$ conidia $\left.\mathrm{cm}^{-2}\right)$ (Figure 2C). Furthermore, Aolatg1 deletion caused a reduction in the spore germination rate: $29.0 \%, 47.6 \%$, and $53.0 \%$ of the $\Delta$ Aolatg1 mutant spores germinated at 4,8 , and $12 \mathrm{~h}$, respectively, whereas $43.5 \%, 56.4 \%$, and $78.4 \%$ of the WT strain spores germinated at the same time points (Figure 2D). However, no obvious differences in the numbers of aerial hyphae and conidiophores, spore yield, and spore germination rate were observed between the WT strain and $\Delta$ Aolatg13 mutant.
The transcript levels of ten sporulation-related genes were determined in the WT, $\Delta$ Aolatg1, and $\Delta$ Aolatg13 mutant strains using qRT-PCR at different growth stages. The transcript levels of $f l b A$ and medA were remarkably upregulated in the $\Delta$ Aolatg1 mutant on days 3 and 5 , and the remaining eight analyzed genes, including abaA, brlA, flbC, fluG, nsdD, velB, vosA, and wet $A$, were downregulated on day 7 (Figure 2E). In the $\Delta$ Aolatg13 mutant, $f l b C, f l b A$, and medA were significantly upregulated on day 3, five genes ( $b r l A, f l b C, f l u G, v e l B$, and wet $A$ ) were downregulated on day 5, and only one gene, $f l b C$ was downregulated on day 7 (Figure 2F).

\section{Aolatg1 and Aolatg13 Are Involved in Stress Resistance}

The stress response of fungal strains was evaluated on TG plates. We observed that deletion of Aolatg1 and Aolatg13 altered sensitivity to oxidative and osmotic agents. For example, deletion of Aolatg1 and Aolatg13 increased RGI by oxidative agents. In particular, in the presence of $5 \mathrm{mM} \mathrm{H}_{2} \mathrm{O}_{2}$, the RGI values of the $\Delta$ Aolatg1 (45.9\%) and $\Delta$ Aolatg13 (48.7\%) mutant strains were higher than that of the WT strain (34.5\%), although no significant differences were noted at 10 and $15 \mathrm{mM} \mathrm{H}_{2} \mathrm{O}_{2}$ (Figures 3 A, B). Further, the $\Delta$ Aolatg1 and $\Delta$ Aolatg13 mutants had higher RGI values in the presence of several menadione concentrations (0.04-0.08 $\mathrm{mM}$ and 0.06-0.08 mM, respectively) compared to the RGI value of the WT strain (Figures $\mathbf{3 A}, \mathbf{C}$ ).

\section{A}
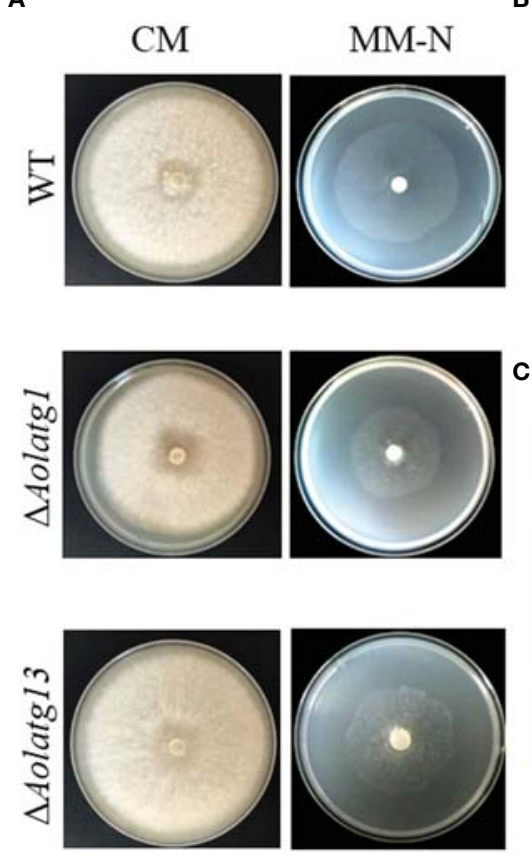

B

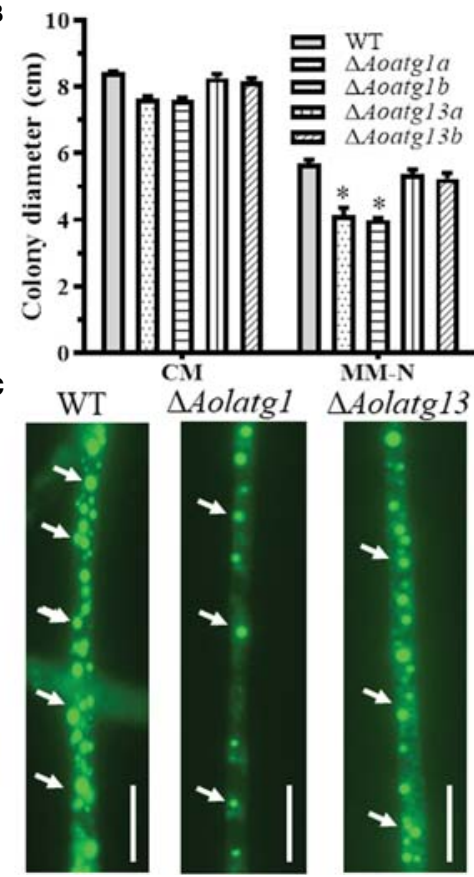

FIGURE 1 | Comparison of mycelial growth in the WT and mutant strains of Arthrobotrys oligospora on CM and MM-N plates. (A) Colony morphology of fungal strains incubated on $\mathrm{CM}$ and $\mathrm{MM}-\mathrm{N}$ plates at $28^{\circ} \mathrm{C}$ for 6 days. (B) Colony diameters of fungal strains incubated on $\mathrm{CM}$ and $\mathrm{MM}-\mathrm{N}$ plates at $28^{\circ} \mathrm{C}$ for 6 days. Data are presented as the mean \pm standard deviation. Statistical significance of differences between mutant strains and WT strain is indicated as follows: ${ }^{*} P<$ 0.05 (Tukey's HSD). (C) Comparison of lipid droplets (LDs) in the WT and mutant strains. The arrows indicate LDs stained with BODIPY staining solution. Scale bars $=10 \mu \mathrm{m}$. 

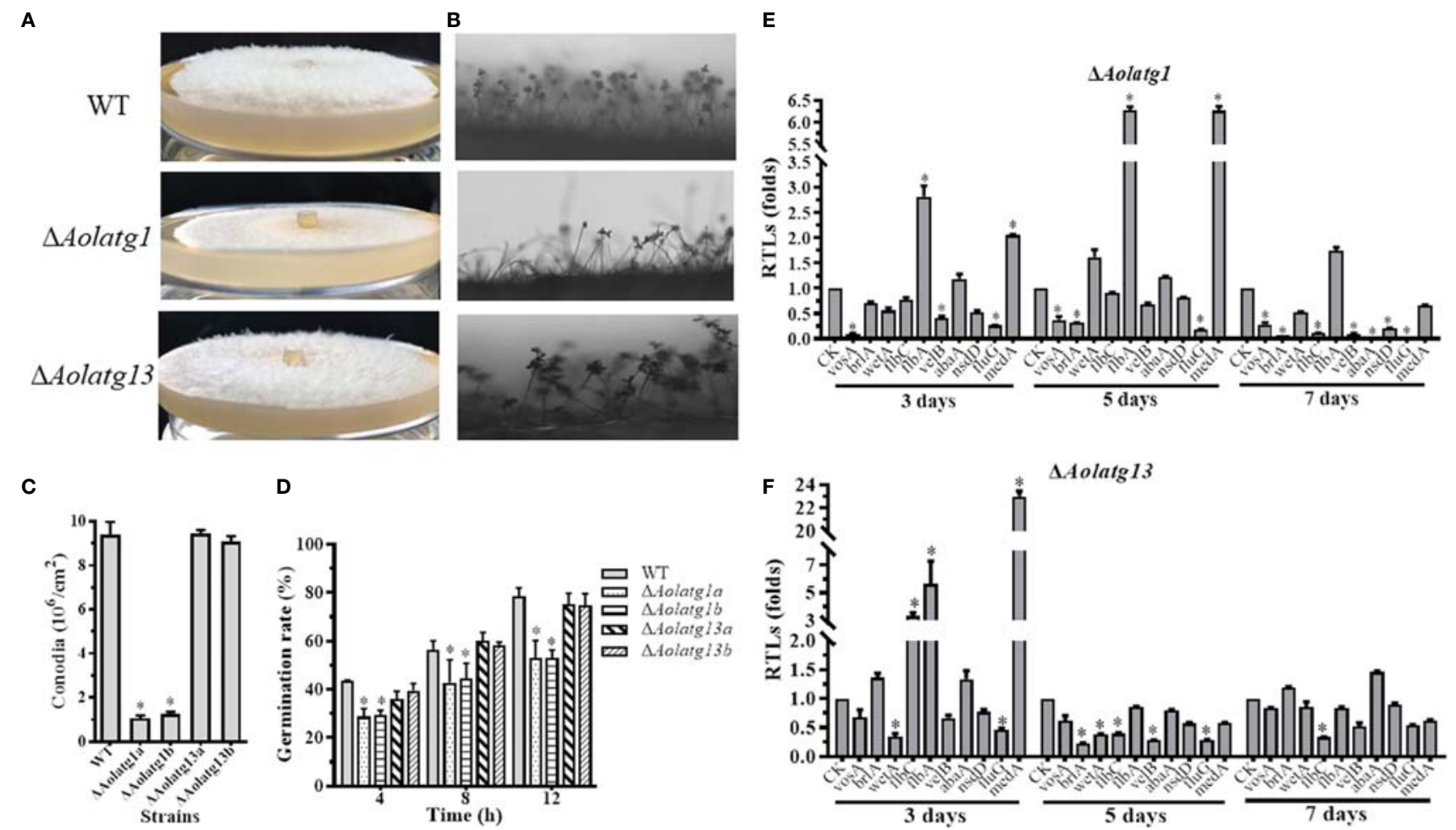

FIGURE 2 | Comparison of conidiation and transcript levels of sporulation-related genes in the WT and mutant strains of $A$. oligospora. (A) Observation of aerial hyphae in the WT and mutant strains. (B) Observation of conidiophores in the WT and mutant strains. (C) Comparison of conidia yields in the WT and mutant strains. (D) Comparison of the germination rate in the WT and mutant strains. (E) The relative transcription levels (RTLs) of the sporulation-related genes in the WT and $\triangle$ AolATG1 mutant strain. (F) The RTLs of sporulation-related genes in the WT and $\triangle$ AolATG13 mutant strain. RTLs of the sporulation-related genes were assessed by comparing the levels of their transcription in the mutant strain with that of the WT strain. CK is the standard used in statistical analysis of the RTL of each gene in the deletion mutant compared to that in the WT strain under any given condition. Data are presented as the mean \pm standard deviation. Statistical significance of differences between mutant strains and WT strain is indicated as follows: ${ }^{\star} P<0.05$ (Tukey's HSD).

In addition, deletion of Aolatg1 and Aolatg13 altered sensitivity to osmotic agents. The $\Delta$ Aolatg13 mutant had a higher RGI value at $0.2-0.3 \mathrm{M} \mathrm{NaCl}$ compared to that of the WT strain, whereas the sensitivity of the $\Delta$ Aolatg1 mutant to $\mathrm{NaCl}$ was not changed significantly (Supplementary Figure S4A, B). Growth of both mutants was more strongly inhibited by sorbitol $(0.5 \mathrm{M}$ for $\Delta$ Aolatg13 and $0.3 \mathrm{M}$ for $\Delta$ Aolatg1) compared to the effect of sorbitol on the WT strain (Supplementary Figure S4A, C).

\section{Aolatg1 and Aolatg13 Regulate Autophagosome Formation}

To probe the effect of Aolatg1 and Aolatg13 deletion on autophagy, we constructed a GFP-Atg8 fusion protein and expressed it in the WT, $\Delta$ Aolatg1, and $\Delta$ Aolatg13 mutant strains. The WT and mutant strains were cultured in the CM medium for $24 \mathrm{~h}$, and then transferred to the MM-N medium and incubated for $6 \mathrm{~h}$. GFP-Atg8 signals were observed in the vacuoles of the hyphae in the WT strain (Figure 4A), whereas punctate GFP signals were observed near the vacuoles in the $\triangle$ Aolatg1 and $\triangle$ Aolatg13 mutants. We then used TEM to visualize autophagosomes and confirmed that when fungal strains were cultured in the MM-N medium for $6 \mathrm{~h}$, autophagosomes were clearly observed in the vacuoles of the WT strain, whereas there were few autophagosomes or autophagosome-like structures in the vacuoles of the $\Delta$ Aolatg1 and $\Delta$ Aolatg13 mutants (Figure 4B).

\section{Aolatg1 Regulates Trap Formation and Pathogenicity}

The WT and mutant strains were incubated on water agar plates at $28^{\circ} \mathrm{C}$, followed by the addition of nematodes to induce trap formation. The WT and $\Delta$ Aolatg13 mutant strains produced more traps than the $\Delta$ Aolatg1 mutant (Figure 5A). At 12, 24, and $36 \mathrm{~h}$, the WT strain produced $1,893,2,953$, and 3,804 traps per plate, respectively. The $\Delta$ Aolatg 13 mutant generated 1,606, 2,690, and 3,998 traps per plate at the same time points, whereas the $\Delta$ Aolatg1 mutant produced only 582, 756, and 971 traps, respectively (Figure 5B). Accordingly, upon the formation of traps, $27 \%, 59.7 \%$, and $99.5 \%$ of nematodes were captured by the WT strain at 12,24 , and $48 \mathrm{~h}$, respectively, and for the $\Delta$ Aolatg 13 mutant the corresponding fractions were similar: $26.7 \%, 56.7 \%$, and $90.9 \%$. In contrast, only $19.5 \%, 41 \%$, and $50 \%$ nematodes were captured by the $\Delta$ Aolatg 1 mutant at the corresponding time points (Figure 5C). 


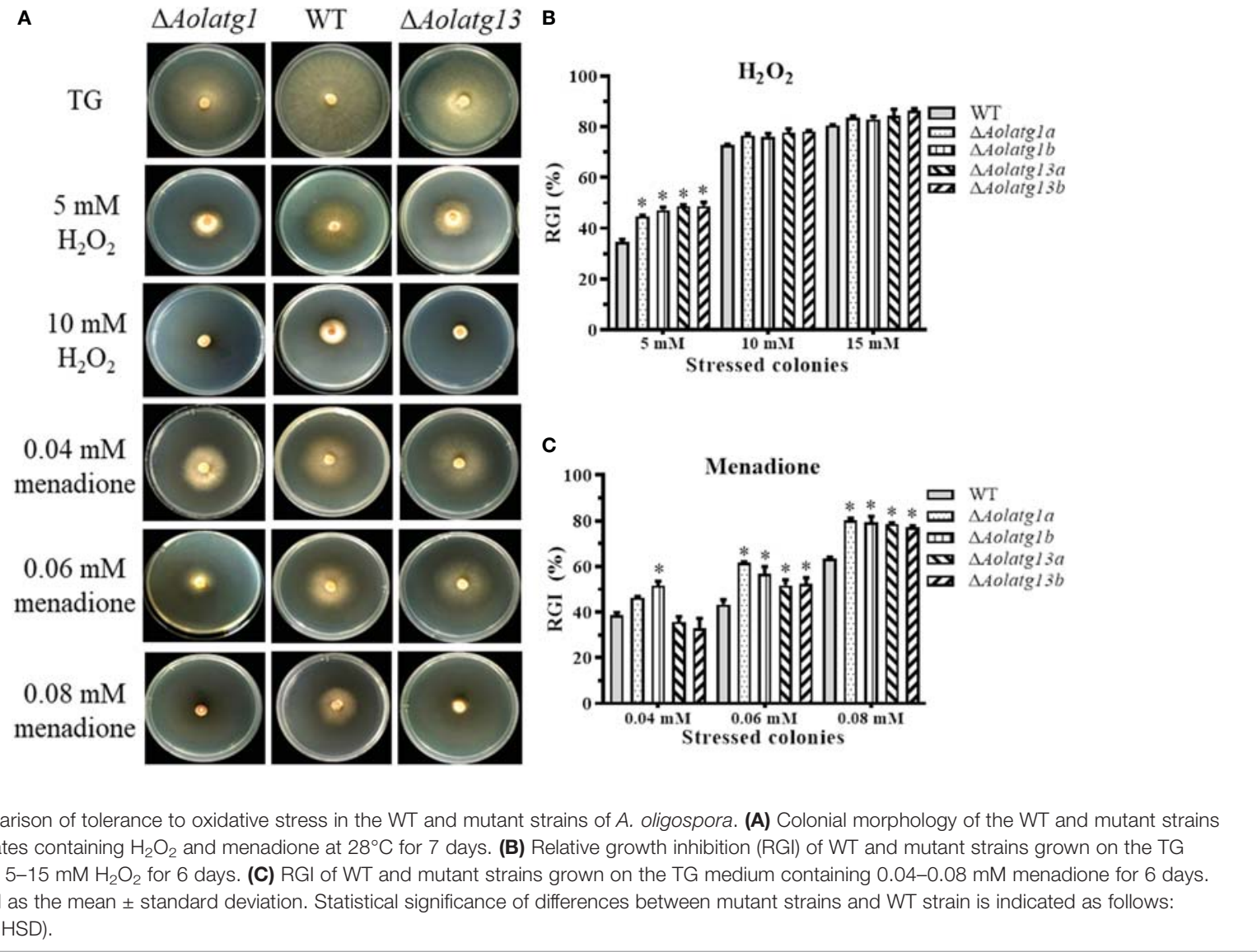

\section{Transcriptomic Analysis of the WT and $\Delta$ Aolatg1 Mutant Strains}

The transcriptomic profiles of the WT and $\Delta$ Aolatg1 mutant strains were compared by RNA-seq. The raw and clean RNA-seq reads are shown in Supplementary Table S4. The percentage of phred-like quality scores at the Q30 level ranged from 91.5\% to $92.9 \%$, and the GC content ranged from $47.7 \%$ to $50.3 \%$ (Supplementary Table S4). The principal component analysis results showed that the WT and $\Delta$ Aolatg1 mutant strains were located in different quadrants, suggesting that their transcription profiles were significantly different, whereas the independent samples at each time point were in close proximity, indicating high similarity and good reproducibility of the three repeats (Supplementary Figure S4).

A total of 2,545 and 4,103 DEGs were identified at 0 and $24 \mathrm{~h}$, respectively, between the $\mathrm{WT}$ and $\Delta$ Aolatg1 mutant strains, whereas expression levels of 1,632 genes were similar at these time points (Figure 6A). At 0 h, 1,410 genes were upregulated and 1,135 were downregulated in the WT compared to the $\Delta$ Aolatg1 mutant (Figure 6B). The upregulated genes were enriched in 164 GO terms and 29 KEGG pathways (Supplementary Figure S5B, C). In particular, membrane (intrinsic/integral component of membrane, membrane part, and membrane), catalytic activity, and ion binding were the highly enriched terms in the GO analysis (Supplementary Figure S6A). In the KEGG analysis, the following metabolic pathways were highly enriched: carbohydrate metabolism, amino acid metabolism, and lipid metabolism (Supplementary Figure S5C and Figure 6C). Fold sorting and degradation, and translation were enriched in genetic information processing; transport and catabolism and cell growth and death were enriched in cellular processes (Figure 6E). The downregulated genes were enriched in $132 \mathrm{GO}$ terms and 24 KEGG pathways (Supplementary Figure S5B). The catalytic activity was highly enriched in the GO analysis (Supplementary Figure S6B). In the KEGG analysis, metabolic pathways and biosynthesis of secondary metabolites were highly enriched, such as carbohydrate metabolism, amino acid metabolism, lipid metabolism, energy metabolism, nucleotide metabolism, and metabolism of terpenoids and polyketides (Supplementary Figure S7).

After induction with nematodes for $24 \mathrm{~h}, 1,976$ genes were upregulated and 2,127 were downregulated in the WT strain compared to their levels in the $\Delta$ Aolatg1 mutant (Figure 6B). The upregulated genes were enriched in 97 GO terms and 26 KEGG pathways (Supplementary Figure S5B, C), membranes were highly enriched in GO terms (Supplementary Figure S6C), whereas amino sugar and nucleotide sugar metabolism, starch and sucrose metabolism, MAPK signaling pathway, autophagy, ubiquitin- 

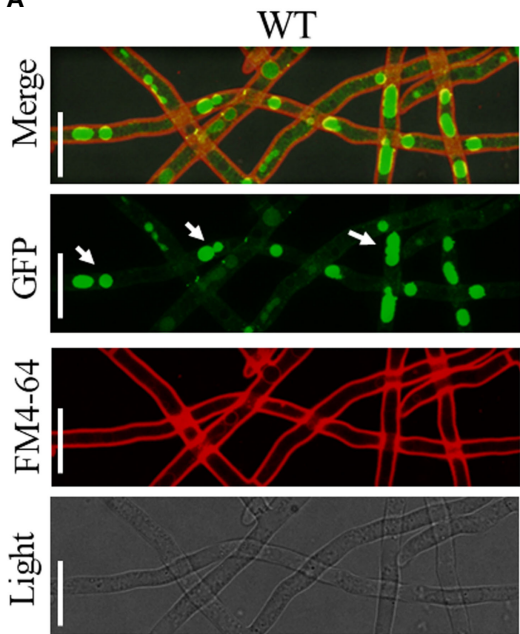

B

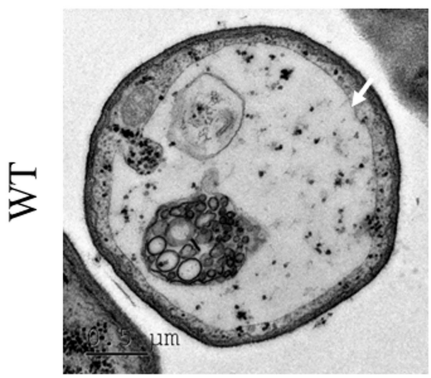

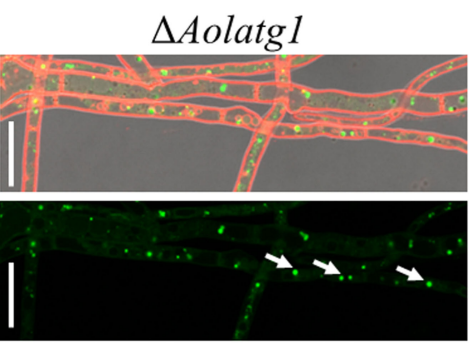
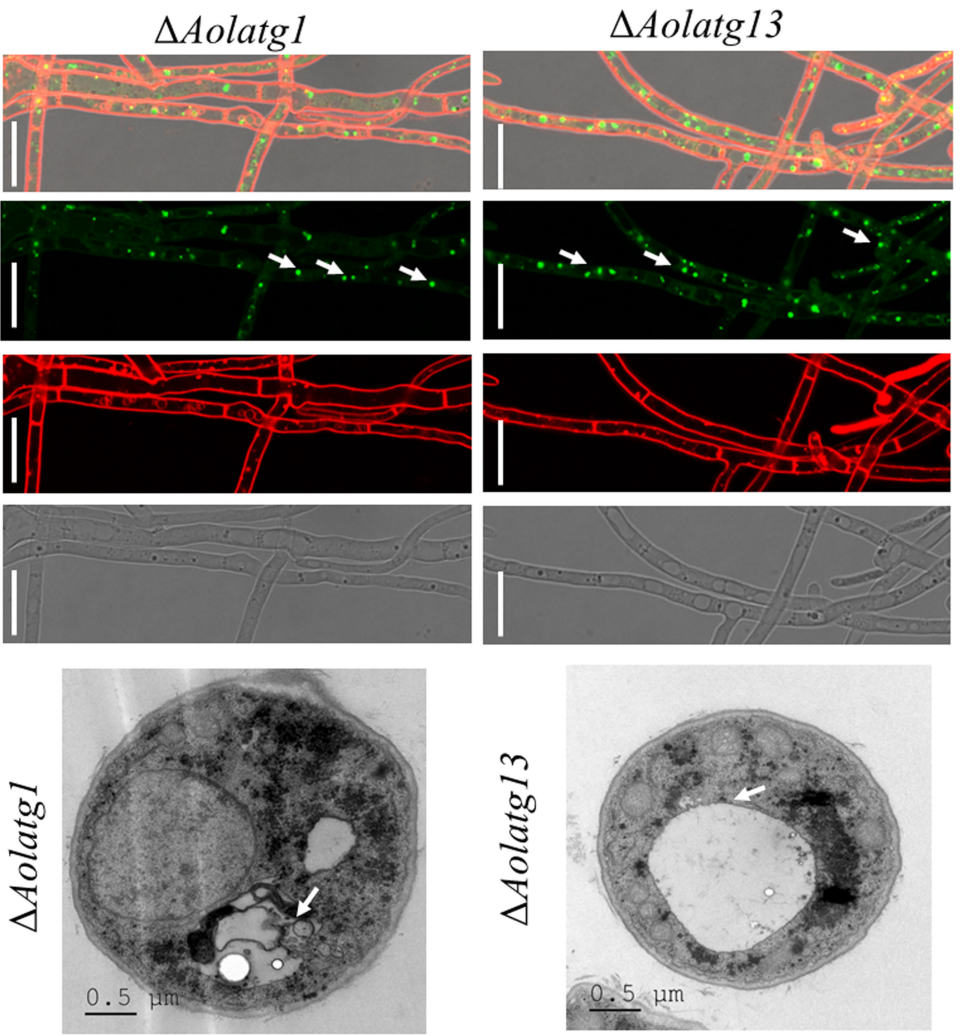

FIGURE 4 | Comparison of the autophagy process in the WT and mutant strains of $A$. oligospora. (A) Expression of the GFP-Atg8 protein in the WT and mutant strains. The A. oligospora strains were grown in the liquid $\mathrm{CM}$ medium at $28^{\circ} \mathrm{C}$ for $24 \mathrm{~h}$, and then transferred to the liquid MM-N medium for $6 \mathrm{~h}$. The vacuoles were stained by FM4-64 and examined by fluorescence microscopy. White arrow: GFP signals. Scale bars = $10 \mu \mathrm{m}$. (B) The vacuoles of hyphal cells were observed using transmission electron microscopy. Arrows indicate the vacuole. Scale bars $=0.5 \mu \mathrm{m}$.

mediated proteolysis, and SNARE interaction in vesicular transport were enriched in KEGG pathways (Figure 6D), and carbohydrate metabolism, amino acid metabolism, and lipid metabolism were highly enriched in metabolism (Figure 6F). The downregulated genes were enriched in 198 GO terms and 28 KEGG pathways (Supplementary Figure S5B, C), cellular components were highly enriched in GO terms, such as the cytoplasm, ribosome, mitochondrion, and non-membrane-bounded organelle (Supplementary Figure S6D). In the KEGG pathway analysis, metabolic pathways and biosynthesis of secondary metabolites were enriched; with carbohydrate metabolism, amino acid metabolism, energy, and lipid metabolism being the particularly highly enriched processes (Supplementary Figure S8).

The comparison of the transcript levels of the genes associated with trap formation (Table 1) revealed that the expression of $f l b A$ was significantly increased by 5.69 folds in $\triangle$ Aolatg1 mutant compared to the WT at $24 \mathrm{~h}$ post-induction (hpi) with nematode, and its expression was unaltered at $0 \mathrm{~h}$. The expression of hog1 in the $\Delta$ Aolatg1 mutant was augmented by 1.92 folds at 24 hpi. However, the expression levels of slt 2 and fus3 remained unperturbed in both WT and $\Delta$ Aolatg1 mutant strains. The atg 8 expression level increased by 2.72 and 2.50 folds in the $\Delta$ Aolatg1 mutant at $0 \mathrm{~h}$ and $24 \mathrm{hpi}$, respectively. Similarly, the expression of $u b r 1$ was elevated by 2.14 and 1.83 folds in the $\Delta$ Aolatg1 mutant at $0 \mathrm{~h}$ and $24 \mathrm{hpi}$, respectively. Additionally, the expression levels of genes involved in oxidative stress response was also evaluated (Table 1). The comparative analysis demonstrated that the expression of genes per and nox 1 was enhanced by 2.80 and 3.71 folds, respectively in the $\Delta$ Aolatg1 mutant compared to that in the WT at $24 \mathrm{hpi}$,. The expression of cat 2 was enriched by 4.15 and 12.84 folds in the $\Delta$ Aolatg 1 mutant at $0 \mathrm{~h}$ and $24 \mathrm{hpi}$, respectively. Similarly, the expression of sod-2, a gene encoding a superoxide dismutase was increased by 2.0 and 5.81 folds in the $\Delta$ Aolatg 1 mutant at $0 \mathrm{~h}$ and $24 \mathrm{hpi}$, respectively. In contrast, the expression of the cat gene (AOL_s00173g374) was decreased by 2.08 folds in the $\Delta$ Aolatg1 mutant at $0 \mathrm{~h}$. However, the expression of another cat gene (AOL_s00188g243) was reduced by 4.76 and 5.73 folds in the $\Delta$ Aolatg1 mutant at $0 \mathrm{~h}$ and $24 \mathrm{hpi}$, respectively. In addition, the expression of noxR was also decreased by 2.54 folds in the $\Delta$ Aolatg1 mutant at $24 \mathrm{hpi}$.

\section{DISCUSSION}

Autophagy is an evolutionarily conserved physiological process in eukaryotic cells that regulates programmed cell fate, tissue and 
A
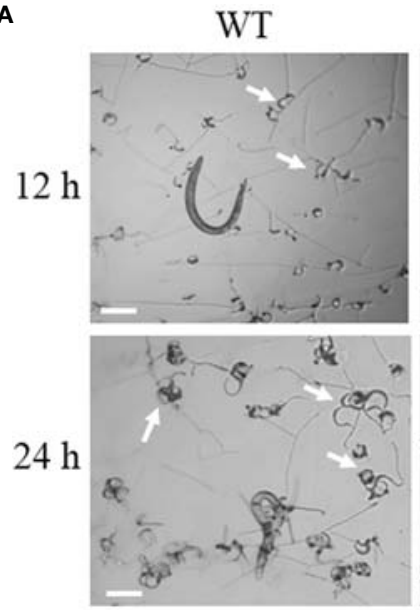

$48 \mathrm{~h}$

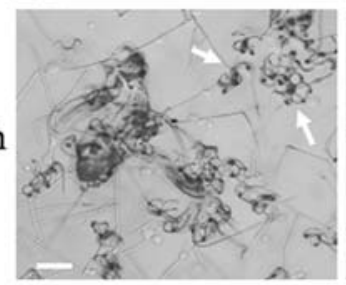

$\Delta$ Aolatg1
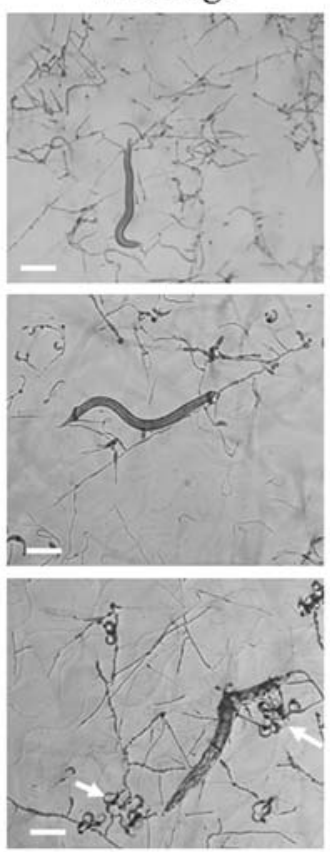

$\Delta$ Aolatg13
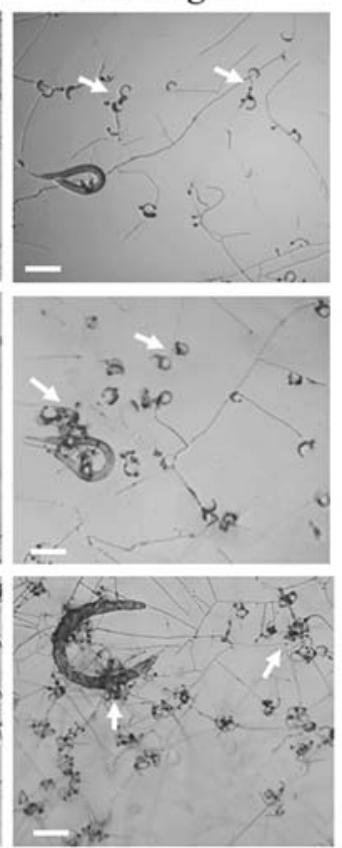

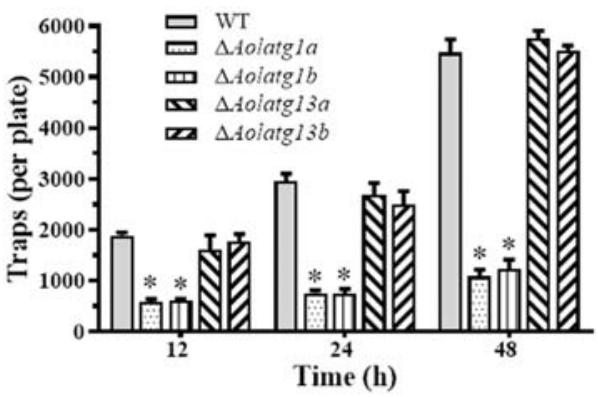

C

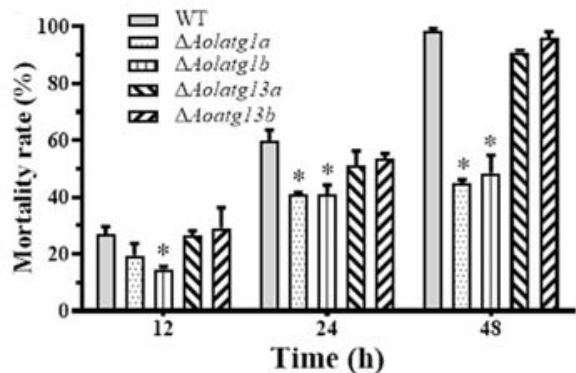

FIGURE 5 | Comparison of trap formation and nematicidal activity in the WT and mutant strains of $A$. oligospora. (A) Traps (indicated by white arrows) were induced by nematodes at 12,24 , and $48 \mathrm{~h}$. Scale bars $=100 \mu \mathrm{m}$. (B) Traps produced by the WT and mutant strains at 12, 24, and $48 \mathrm{~h}$. (C) Nematode mortality rate (\%) at 12,24 , and $48 \mathrm{~h}$. Data are presented as the mean \pm standard deviation. Statistical significance of differences between mutant strains and $\mathrm{WT}$ strain is indicated as follows: ${ }^{\star} P<0.05$ (Tukey's HSD).

cellular remodeling, and development (Pollack et al., 2009). Atg1 and Atg13 are core Atg proteins involved in the initial nucleation step of the phagophore formation (Itakura and Mizushima, 2010). Recently, Atg1 has been shown to be involved in the Cvt pathway and to play an essential role in the regulation of mycelial growth, conidiation, and virulence in filamentous fungi (Zhu et al., 2018; Ying and Feng, 2019). In this study, orthologs of Atg1 and Atg13 were characterized in the typical NT fungus A. oligospora, and their roles in autophagy, asexual development, trap formation, and nematode predation were comprehensively compared.

We used the GFP-Atg8 fusion protein to visualize autophagy and observed that in the WT strain, GFP signals accumulated in the vacuole, whereas in the $\Delta$ Aolatg1 and $\Delta$ Aolatg13 mutants, the majority of GFP signals were dispersed outside the vacuole in the hyphae, suggesting that the absence of the Aolatg1 and Aolatg13 genes blocked the autophagy pathway. Similar results have been reported for other fungi. For example, autophagic bodies were observed in the vacuoles of the WT strain of Fusarium graminearum, whereas no autophagic bodies or a small number of autophagosome-like structures were observed in the vacuoles of a $\Delta$ Fgatg1 mutant (Lv et al., 2017). In A. oryzae, AoAtg1 is essential for nonselective autophagy and the Cvt pathway (Yanagisawa et al., 2013), and only a slight accumulation of EGFP-AoAtg8 in the vacuoles of the $\Delta$ Aoatg13 mutant was observed (Kikuma and
Kitamoto, 2011). Deletion of Atg1 abolished autophagosome accumulation in the vacuoles of carbon-starved Ustilago maydis cells (Nadal and Gold, 2010). In addition, deletion of Bcatg1 inhibited autophagosome accumulation in the vacuoles of nitrogen-starved B. cinerea cells (Ren et al., 2017). Similarly, autophagy was blocked in the $\Delta$ Mgatg1 (Liu et al., 2007) and $\Delta$ Bbatg1 mutants (Ying et al., 2016). These findings suggest that orthologs of Atg1 and Atg13 are indispensable for autophagy in various fungi.

Mycelial growth was impaired in the absence of Aolatg1 and Aolatg13 compared to that in the WT strain. The colony size and aerial mycelia of the $\Delta$ Aolatg1 mutant were remarkably lower, whereas the mycelial growth of the $\Delta$ Aolatg13 mutant was slightly reduced on the PDA, TG, and CMY media, but not on the CM and MM-N media. Moreover, deletion of Aolatg1 and Aolatg13 caused a remarkable reduction in LDs in hyphal cells. In A. oryzae, deletion of Aoatg1 and Aoatg13 did not affect colony size, but developed aerial hyphae were scarcely observed in the $\Delta$ Aoatg1 mutant (Yanagisawa et al., 2013). Deletion of Fgatg1 reduced the hyphal growth of $F$. graminearum (Lv et al., 2017), but colonies of the $\Delta$ Fgatg13 mutant were the same as those of the WT strain when cultured on PDA plates (Lv et al., 2017). In B. cinerea, the mycelial radial growth rate of the $\Delta B$ catg1 mutant was broadly similar to that of the WT, but the former produced significantly fewer aerial hyphae with more of 
A WT vs $\Delta$ Aolatgl

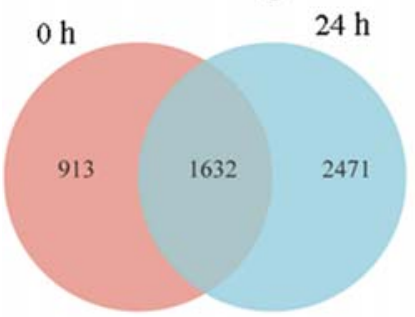

C

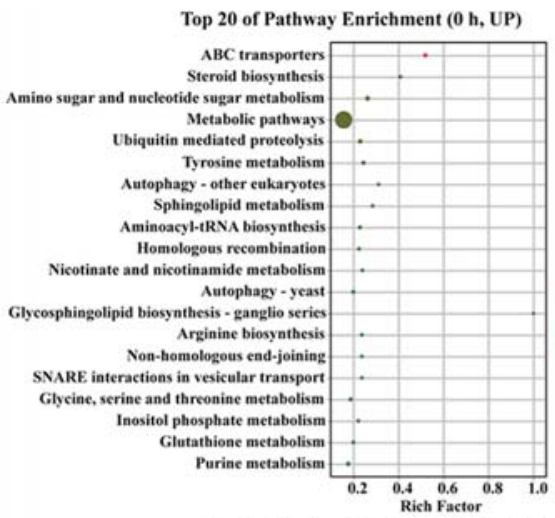

Top 20 of Pathway Enrichment (24 h, UP)

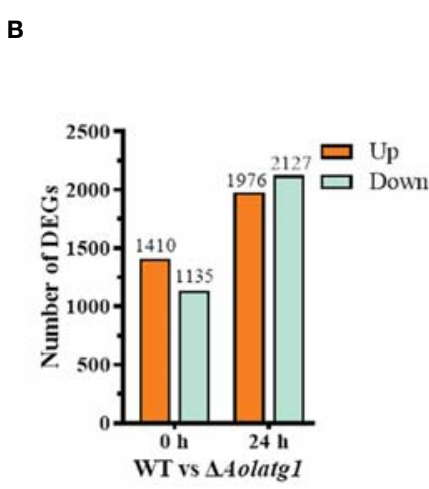

D

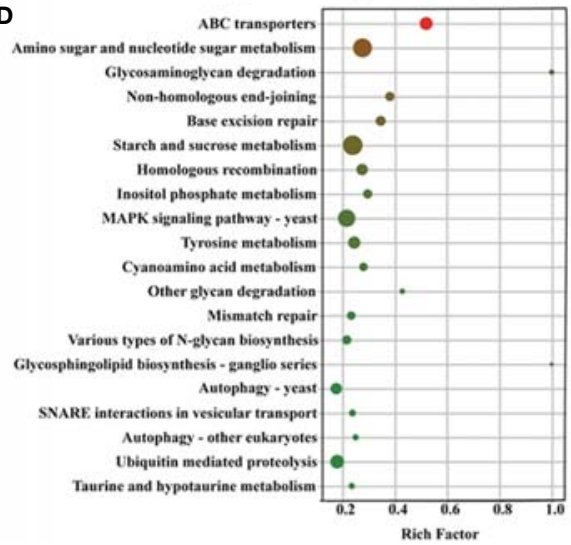

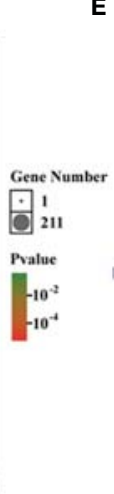
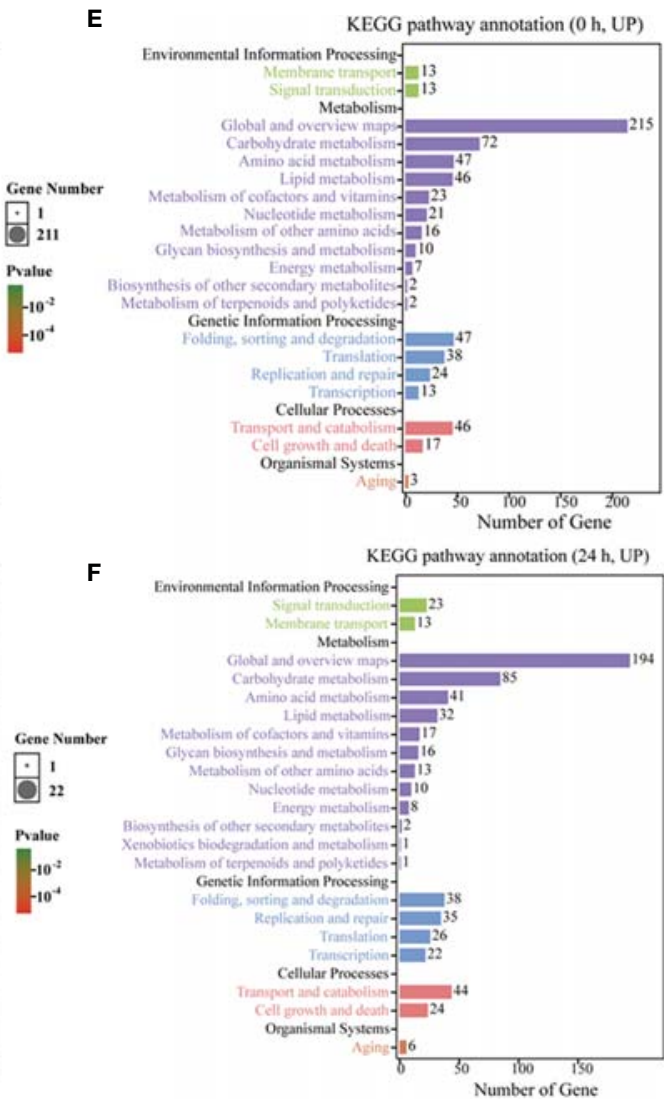

$\mathbf{F}$

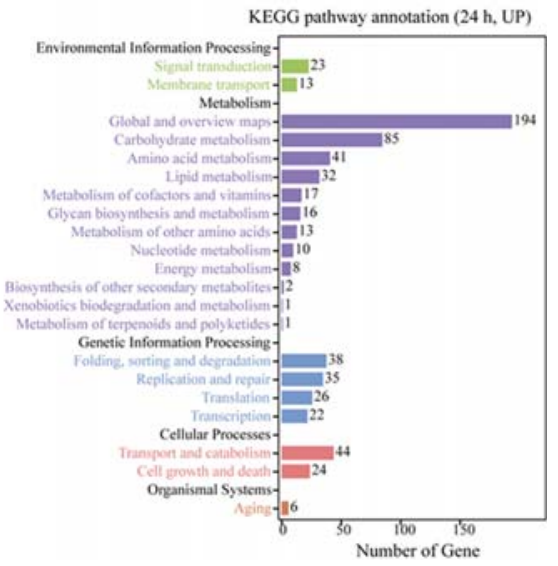

FIGURE 6 | Comparison of differentially expressed genes (DEGs) between WT and the $\triangle$ Aolatg1 mutant strain of A. oligospora. (A) Venn analysis of DEGs. (B) The number of upregulated and downregulated DEGs in the WT versus the $\Delta$ Aolatg1 mutant strains. (C) The top 20 pathways that are upregulated in the WT strain compared to the $\Delta$ Aolatg1 mutant strain at $0 \mathrm{~h}$. (D) The top 20 pathways that are upregulated in the WT strain compared to the $\Delta$ Aolatg 1 mutant strain at $24 \mathrm{~h}$. (E) Annotation of the upregulated KEGG pathways in the WT strain compared to the pathways in $\triangle$ Aolatg1 mutant at 0 h. (F) Annotation of the upregulated KEGG pathways in the WT strain compared to the pathways in $\Delta$ Aolatg1 mutant at $24 \mathrm{~h}$.

them being fused (Ren et al., 2017). As in the $\Delta$ Bcatg1 mutant of B. cinerea, the $\Delta$ Mgatg1 mutant had sparse aerial hyphae on both $\mathrm{CM}$ and MM plates, although growth of its colonies was not affected (Liu et al., 2007). These findings suggest that orthologs of Atg1 play an essential role in mycelial development, especially in the growth of aerial hyphae, whereas Atg13 has only a minor role in these processes.

Deletion of Aolatg1 caused a remarkable reduction in spore yield and spore germination rate, whereas deletion of Aolatg13 did not affect the sporulation of A. oligospora. Transcription of several sporulation-related genes, such as $f l u G, a b a A, b r l A$, and $v e l B$, was significantly downregulated in the $\Delta$ Aolatg1 mutant compared to the WT strain on day 7 , whereas in the $\Delta$ Aolatg13 mutant, transcription levels of these genes showed no obvious change. Similarly, deletion of Aoatg1 strongly inhibited the conidiation in $A$. oryzae (Yanagisawa et al., 2013), and the number of conidia was lower in the $\Delta$ Aoatg13 mutant than in the WT strain (Kikuma and Kitamoto, 2011). In B. cinerea, the $\Delta B$ catg1 mutant produced significantly fewer conidia than the WT strain, and most conidia showed an aberrant shape with many vacuoles (Ren et al., 2017). In F. graminearum, the conidiation was significantly reduced in the $\Delta$ Fgatg1 and $\Delta$ Fgatg13 mutants (Lv et al., 2017). Conidiogenesis in the $\Delta$ Mgatg1 mutant was reduced significantly, and the conidia of $\Delta$ Mgatg1 mutants germinated more slowly than those of the WT strain (Liu et al., 2007). Moreover, a mutant with disrupted Bbatg1 had impaired conidial yield and conidial germination under starvation stress (Ying et al., 2016). These findings suggest that Atg1 plays a conserved and important role in the conidiation of many filamentous fungi, whereas the role of Atg13 in conidiation varies among fungi.

Apart from mycelial growth and conidiation, deletion of Aolatg1 and Aolatg13 impaired stress resistance, as the $\Delta$ Aolatg1 and $\Delta$ Aolatg13 mutants were more sensitive to oxidative stress caused by menadione and $\mathrm{H}_{2} \mathrm{O}_{2}$ than the WT strain. Furthermore, the $\Delta$ Aolatg13 mutant was also sensitive to osmotic pressure $(\mathrm{NaCl}$ and sorbitol). It has been shown that deletion of Aolatg4 and Aolatg5 in A. oligospora also altered sensitivity to oxidative and osmotic stresses (Zhou et al., 2020; Zhou et al., 2021). In $B$. bassiana, the $\Delta B b a t g 8$ mutant exhibited enhanced sensitivity to 
TABLE 1 | Transcriptional response to Aolatg1 deletion by the genes involved in trap formation and oxidative stress response in comparative transcriptome analysis.

\begin{tabular}{|c|c|c|c|c|c|c|}
\hline \multirow[t]{3}{*}{ Locus } & \multirow[t]{3}{*}{ Function annotation } & \multicolumn{4}{|c|}{ Expressional levels } & \multirow[t]{3}{*}{ References } \\
\hline & & \multicolumn{2}{|c|}{ TPM-0 h } & \multicolumn{2}{|c|}{ TPM-24 h } & \\
\hline & & WT & $\Delta$ Aolatg1 & WT & $\Delta$ Aolatg13 & \\
\hline \multicolumn{7}{|c|}{ Genes involved in trap formation and pathogenicity } \\
\hline AOL_s00215g516 & flbA, developmental regulator & 109.05 & 101.09 & 29.29 & 166.74 & (Ma et al., 2021) \\
\hline AOL_S00215g7 & ras2, Ras family & 480.70 & 601.97 & 130.02 & 75.16 & (Yang et al., 2021) \\
\hline AOL_s00054g446 & rab-7A, Rab small GTPase & 221.4 & 241.79 & 186.05 & 263.07 & (Yang et al., 2018) \\
\hline AOL_s00054g68 & glo3, Arf GAP & 119.8 & 138 & 100.8 & 127.71 & (Ma et al., 2020) \\
\hline AOL_s00110g154 & fus3, MAP kinase & 89.59 & 86.69 & 119.39 & 109.09 & (Chen et al., 2021) \\
\hline AOL_s00109g23 & hog1, MAP kinase & 146.71 & 148.25 & 137.99 & 265.83 & (Kuo et al., 2020) \\
\hline AOL_s00173g235 & slt2, MAP kinase & 169.76 & 150.98 & 191.17 & 186.58 & (Zhen et al., 2018) \\
\hline AOL_s00083g25 & stuA, APSES transcription factor & 51.04 & 37.8 & 48.2 & 45.78 & (Xie et al., 2019) \\
\hline AOL_s00007g534 & atg8, autophagy-related protein 8 & 769.28 & 2096.53 & 596.99 & 1493.93 & (Chen et al., 2013) \\
\hline AOL_s00112g56 & hex 1 , woronin body major protein & 1590.13 & 2597.71 & 3231.56 & 2343.38 & (Liang et al., 2017) \\
\hline AOL_s00080g296 & ubr1, E3 ubiquitin-protein ligase & 40.98 & 87.82 & 25.94 & 47.47 & (Zhang et al., 2021) \\
\hline AOL_s00054g811 & velB, developmental regulator & 119.08 & 73.47 & 103.64 & 62.76 & (Zhang et al., 2019) \\
\hline \multicolumn{7}{|c|}{ Genes involved in oxidative stress response } \\
\hline AOL_s00109g143 & per, peroxidase & 79.47 & 113.59 & 70.81 & 198.26 & (Zhu et al., 2013) \\
\hline AOL_s00173g374 & cat, catalase & 304.47 & 146.51 & 355.82 & 304.33 & (Michán et al., 2003) \\
\hline AOL_s00188g243 & cat, catalase & 1.19 & 0.25 & 1.49 & 0.26 & (Wang et al., 2007) \\
\hline AOL_s00006g411 & cat2, catalase & 1.48 & 6.14 & 3.00 & 38.53 & (Sun et al., 2019) \\
\hline AOL_s00193g69 & nox-1, NADPH oxidase & 120.89 & 178.27 & 67.51 & 250.78 & (Li et al., 2017) \\
\hline AOL_s00007g557 & nox-2, NADPH oxidase & 38.43 & 53.72 & 81.44 & 96.65 & (Cano-Domínguez et al., 2008) \\
\hline AOL_s00054g538 & noxR, NADPH oxidase regulator & 29.19 & 16.85 & 186.17 & 73.22 & (Sun et al., 2019) \\
\hline AOL_s00007g292 & sod, superoxide dismutase & 53.73 & 48.84 & 34.25 & 63.31 & (Zhu et al., 2013) \\
\hline AOL_s00054g687 & sodB, superoxide dismutase & 465.97 & 289.02 & 426.03 & 394.27 & (Zhu et al., 2013) \\
\hline AOL_s00170g93 & sod-2, superoxide dismutase & 603.01 & 1206.07 & 359.61 & 2089.07 & (Zhu et al., 2013) \\
\hline
\end{tabular}

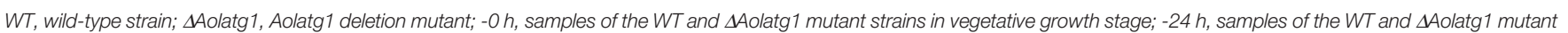
strains after induced with nematodes for $24 \mathrm{~h}$. Locus numbers and function were annotated according to the A. oligospora genome assembly (https://www.ncbi.n/m.nih.gov/). TPM, transcripts per kilobase million.

oxidative stress, whereas the $\Delta B b a t g 1$ mutant did not (Ying et al., 2016). These results show that the autophagy pathway is also involved in regulating the fungal stress response.

Several studies have established that the asexual development of fungi is correlated to oxidative stress response. This is validated by the observation that $\Delta$ cat-3 mutant of Neurospora crassa produces six times more aerial hyphae and conidia compared to the WT strain (Michán et al., 2003). Moreover, the depletion of cat-1 resulted in a significant reduction in the rate of conidial germination (Wang et al., 2007). Recently, a Zn(II)2Cys6-type transcription factor, ADA-6 was identified in N. crassa. Characterization of ada- 6 revealed that its deletion impaired conidial production and induced female sterility. In addition, RNA-seq analysis demonstrated that ADA-6 modulates the transcription of cat-3 and other genes participating in the production of reactive oxygen species during conidiation (Sun et al., 2019). In this study we found that the expressions of several genes associated with oxidative stress response were altered. The cat-2 and sod-2 genes were significantly enhanced in the $\Delta$ Aolatg1 mutant compared to the WT strain at 0 and 24 hpi. However, noxR and the other two cat genes were downregulated in the $\Delta$ Aolatg1 mutant. These findings suggest that defect in conidiation of $\Delta$ Aolatg1 mutant might be connected to the oxidative stress response.

Trap formation in NT fungi is a complex cellular process that was suppressed by deletion of Atg4, Atg5, and Atg8 orthologs, which suggested that autophagy plays an important role in trap development in A. oligospora (Chen et al., 2013; Zhou et al., 2020; Zhou et al., 2021). In this study, we characterized the role of Aolatg1 and Aolatg13 in trap formation and nematode predation. Our results showed that trap formation and nematicidal activity were remarkably decreased in the $\Delta$ Aolatg1 mutant. Autophagy has been recently demonstrated to be closely associated with fungal virulence. For example, deletion of Mgatg1 caused lower turgor pressure of the appressorium, and the $\Delta$ Mgatg1 mutant lost its ability to penetrate and infect host plants (Liu et al., 2007). However, the lack of Mgatg13 did not have any influence on the pathogenicity of M. oryzae (Kershaw and Talbot, 2009). In F. graminearum, the pathogenicity of the $\Delta$ Fgatg1 and $\Delta$ Fgatg13 mutants was lower than that of the WT strain, as these mutants had decreased abilities to infect wheat spikelets and to spread to new spikelets following the original infection (Lv et al., 2017). In $B$. cinerea, most conidia of the $\Delta B$ catg 1 mutant lost the capacity to form the appressorium infection structure and failed to penetrate the onion epidermis, and pathogenicity assays showed that the virulence of $\Delta B$ catg1 tested in different host plant tissues was drastically impaired (Ren et al., 2017). Moreover, the virulence of the $\Delta$ Bbatg1 mutant was considerably weaker than that of the WT strain, as indicated by lower infectivity in the topical and intrahemocoel injection assays (Ying et al., 2016). These findings suggest that Atg1 plays a conserved and crucial role in the virulence of many pathogenic 
fungi, whereas Atg13 effect on virulence is prominent only in few fungi (e.g., F. graminearum).

Transcriptomic analysis showed that more DEGs were identified after the fungi were induced with nematodes compared to the transcriptomic differences between intact fungi. This finding suggests that many genes were mobilized during trap formation. In the GO analysis, the upregulated genes were highly enriched in the membrane-related terms at $0 \mathrm{~h}$ and $24 \mathrm{~h}$, indicating that membrane trafficking plays a crucial role in autophagy, mycelial growth, and trap formation. In turn, the downregulated genes were enriched in catalytic activity at $0 \mathrm{~h}$ and in cellular components at $24 \mathrm{~h}$, including ribosome, mitochondrion, and organelles, suggesting that there are multiple organelles involved in trap formation. In the KEGG analysis, the upregulated genes were highly enriched in metabolic pathways at $0 \mathrm{~h}$; in contrast, except for metabolism, MAPK signaling pathways, autophagy, and autophagy-related processes such as ubiquitin-mediated proteolysis and ANARE interaction in vesicular transport were also enriched at $24 \mathrm{~h}$. These findings suggest that MAPK pathways and autophagy play an important role in trap formation in A. oligospora. In fact, several MAPK signaling proteins have been proved to regulate trap formation in A. oligospora, such as Slt2 (Zhen et al., 2018; Xie et al., 2021), Hog1 (Chen et al., 2021), and Ime2 (Xie et al., 2020). Autophagyrelated proteins such as AolAtg4 (Zhou et al., 2020), AolAtg5 (Zhou et al., 2021), and AolAtg8 (Chen et al., 2013) have also been shown to influence trap formation in A. oligospora, and deletion of AolAtg1 significantly reduced of the number of traps. The set of downregulated genes was highly enriched in genes relevant to metabolic pathways and biosynthesis of secondary metabolites at $0 \mathrm{~h}$ and $24 \mathrm{~h}$. Moreover, genes involved in lipid metabolism were enriched at $0 \mathrm{~h}$ and $24 \mathrm{~h}$, suggesting that autophagy may regulate lipid metabolism. In M. oryzae, deletion of the Mgatg1 gene influenced the number of lipid bodies, and lipid storage in conidia of the $\Delta M g a t g 5$ mutant was lower than in the WT strain (Liu et al., 2007; Lu et al., 2009). Similarly, LDs accumulation was significantly reduced in the conidia of $\Delta B$ catg1, but the glycerol content was increased in $\Delta B$ catg1 mutant (Ren et al., 2017). In addition, DEGs at $0 \mathrm{~h}$ and $24 \mathrm{~h}$ were enriched in genes involved in energy metabolism. Energy is required for trap development, as evidenced by the fact that deletion of the malate synthase gene led to a defect in trap formation (Zhao et al., 2014). Moreover, DEGs at $0 \mathrm{~h}$ and $24 \mathrm{~h}$ were enriched in genes relevant to metabolism of terpenoids and polyketides, which are involved in the biosynthesis of arthrobotrisins, a special group of metabolites identified in $A$. oligospora and other NT fungi (Anderson et al., 1995; Wei et al., 2011). Recently, 6-methylsalicylic acid, an intermediate in the biosynthesis of arthrosporols produced by NT fungi, was found to be a morphogen for spatiotemporal control of trap formation and a chemoattractant that lured $C$. elegans into fungal colonies (Yu et al., 2021). Therefore, transcriptomic analysis provides a good basis for understanding the mechanisms of mycelial growth, development, and pathogenicity.

G-protein signaling plays an indispensable role in trap formation of $A$. oligospora. There are several evidences corroborating this hypothesis, such as deletion of $f l b A$, which encodes a regulator of G-protein signaling, abrogates trap formation in A. oligospora (Ma et al., 2021). Moreover, hog1 deletion caused a reduction in trap formation and predation efficiency in A. oligospora (Kuo et al., 2020). In this study, we observed that the transcripts of $f l b A$ and hogl were markedly upregulated in $\Delta$ Aolatg1 mutant when induced with nematodes. In addition, the expressions of atg 8 and $u b r 1$ were upregulated in $\Delta$ Aolatg1 mutant during the stages of vegetative growth and trap formation. Atg8 has been shown to be indispensable for trap formation in A. oligospora (Chen et al., 2013). Additionally, the $\Delta u b r 1$ mutant of $A$. oligospora exhibits a substantial reduction in vegetative growth and trap formation (Zhang et al., 2021). Therefore, according to these observations, it is evident that AolAtg1 regulates trap formation and pathogenicity of $A$. oligospora by promoting G-protein signaling and regulating protein ubiquitination.

At present, orthologs of Atg1 have been identified in many different fungi and shown to play conserved and indispensable roles in autophagy, mycelial growth, conidiation, lipid metabolism, and pathogenicity. In contrast, Atg13 has been identified only in a limited number of fungal species, and although it plays an essential role in autophagy, its deletion seemed to influence few if any phenotypic traits in most fungi, with the notable exception of $F$. graminearum. Here, we characterized Atg1 and Atg13 in A. oligospora, a typical NT fungus, and showed that AolAtg1 and AolAtg13 play crucial roles in autophagy, whereas their contributions to mycelial growth, conidiation, trap formation, and nematode predation are different. However, the underlying molecular mechanisms by which AolAtg1 and AolAtg13 regulate diverse phenotypes need to be further investigated using detailed comparative analysis of the transcriptome and by various other methods. Meanwhile, because of the lack of available resistance markers for A. oligospora, we failed to construct a double deletion mutant of Aolatg1 and Aolatg13, which could help to understand the interaction between these two proteins in this fungus. Nonetheless, our results provide a solid basis for further investigation of the roles and regulatory mechanisms of atg genes in the growth, development, and pathogenicity of NT fungi.

\section{DATA AVAILABILITY STATEMENT}

The datasets presented in this study can be found in online repositories. The names of the repository/repositories and accession number(s) can be found below: https://www.ncbi. nlm.nih.gov/genbank/, PRJNA784322.

\section{AUTHOR CONTRIBUTIONS}

JY and K-QZ conceived and designed the study. DZ and YZ conducted the experiments. DZ, YZ, NB, and MX analyzed the data. JY, DZ, and YZ wrote and revised the manuscript. All authors have read and approved the final manuscript. 


\section{FUNDING}

This study was supported by the grants from the National Natural Science Foundation of China (No. 31960556) and the Applied Basic Research Foundation of Yunnan Province (No. 202001BB050004).

\section{ACKNOWLEDGMENTS}

We are grateful to the Microbial Library of the Germplasm Bank of wild species from Southwest China for preserving and

\section{REFERENCES}

Anderson, M. G., Jarman, T. B., and Rickards, R. W. (1995). Structures and Absolute Configurations of Antibiotics of the Oligosporon Group From the Nematode-Trapping Fungus. Arthrobotrys oligospora. J. Antibiot (Tokyo). 48, 391-398. doi: 10.7164/antibiotics.48.391

Bai, N., Zhang, G., Wang, W., Feng, H., Yang, X., Zheng, Y., et al. (2021). Ric8 Acts as a Regulator of G-Protein Signalling Required for Nematode-Trapping Lifecycle of Arthrobotrys Oligospora. Environ. Microbiol. doi: 10.1111/14622920.15735

Cano-Domínguez, N., Alvarez-Delfín, K., Hansberg, W., and Aguirre, J. (2008). NADPH Oxidases NOX-1 and NOX-2 Require the Regulatory Subunit NOR-1 to Control Cell Differentiation and Growth in. Neurospora crassa. Eukaryot Cell. 7, 1352-1361. doi: 10.1128/EC.00137-08

Chen, Y. L., Gao, Y., Zhang, K. Q., and Zou, C. G. (2013). Autophagy is Required for Trap Formation in the Nematode-Trapping Fungus Arthrobotrys oligospora. Environ. Microbiol. Rep. 5, 511-517. doi: 10.1111/1758-2229.12054

Chen, S. A., Lin, H. C., Schroeder, F. C., and Hsueh, Y. P. (2021). Prey Sensing and Response in a Nematode-Trapping Fungus is Governed by the MAPK Pheromone Response Pathway. Genetics 217:iyaa008. doi: 10.1093/genetics/ iyaa008

Cheong, H., Nair, U., Geng, J., and Klionsky, D. J. (2008). The Atg1 Kinase Complex is Involved in the Regulation of Protein Recruitment to Initiate Sequestering Vesicle Formation for Nonspecific Autophagy in Saccharomyces cerevisiae. Mol. Biol. Cell 19, 668-681. doi: 10.1091/mbc.e07-08-0826

Chew, L. H., Lu, S., Liu, X., Li, F. K., Yu, A. Y., Klionsky, D. J., et al. (2015). Molecular Interactions of the Saccharomyces Cerevisiae Atg1 Complex Provide Insights Into Assembly and Regulatory Mechanisms. Autophagy 11, 891-905. doi: 10.1080/15548627.2015.1040972

Dong, B., Liu, X. H., Lu, J. P., Zhang, F. S., Gao, H. M., Wang, H. K., et al. (2009). MgAtg9 Trafficking in Magnaporthe Oryzae. Autophagy 5, 946-953. doi: 10.4161/auto.5.7.9161

Itakura, E., and Mizushima, N. (2010). Characterization of Autophagosome Formation Site by a Hierarchical Analysis of Mammalian Atg Proteins. Autophagy 6, 764-776. doi: 10.4161/auto.6.6.12709

Kamada, Y., Funakoshi, T., Shintani, T., Nagano, K., Ohsumi, M., and Ohsumi, Y. (2000). Tor-Mediated Induction of Autophagy via an Apg1 Protein Kinase Complex. J. Cell Biol. 150, 1507-1513. doi: 10.1083/jcb.150.6.1507

Kershaw, M. J., and Talbot, N. J. (2009). Genome-Wide Functional Analysis Reveals That Infection-Associated Fungal Autophagy is Necessary for Rice Blast Disease. Proc. Natl. Acad. Sci. U.S.A. 106, 15967-15972. doi: 10.1073/ pnas.0901477106

Kikuma, T., and Kitamoto, K. (2011). Analysis of Autophagy in Aspergillus Oryzae by Disruption of Aoatg13, Aoatg4, and Aoatg15 Genes. FEMS Microbiol. Lett. 316, 61-69. doi: 10.1111/j.1574-6968.2010.02192.x

Kroemer, G., and Levine, B. (2008). Autophagic Cell Death: The Story of a Misnomer. Nat. Rev. Mol. Cell Biol. 9, 1004-1010. doi: 10.1038/nrm2529

Kumar, S., Stecher, G., and Tamura, K. (2016). MEGA7: Molecular Evolutionary Genetics Analysis Version 7.0 for Bigger Datasets. Mol. Biol. Evol. 33, 18701874. doi: 10.1093/molbev/msw054

Kuo, C. Y., Chen, S. A., and Hsueh, Y. P. (2020). The High Osmolarity Glycerol (HOG) Pathway Functions in Osmosensing, Trap Morphogenesis and providing experimental strains. We also thank Guo Yingqi (Kunming Institute of Zoology, Chinese Academy of Sciences) for her help with the electron microscopy image analysis.

\section{SUPPLEMENTARY MATERIAL}

The Supplementary Material for this article can be found online at: https://www.frontiersin.org/articles/10.3389/fcimb.2021. 824407/full\#supplementary-material

Conidiation of the Nematode-Trapping Fungus Arthrobotrys Oligospora. J. Fungi 6, 191. doi: 10.3390/jof6040191

Liang, L., Gao, H., Li, J., Liu, L., Liu, Z., and Zhang, K. Q. (2017). The Woronin Body in the Nematophagous Fungus Arthrobotrys Oligospora is Essential for Trap Formation and Efficient Pathogenesis. Fungal Biol. 121, 11-20. doi: 10.1016/j.funbio.2016.08.010

Li, B., and Dewey, C. N. (2011). RSEM: Accurate Transcript Quantification From RNA-Seq Data With or Without a Reference Genome. BMC Bioinf. 12:323. doi: 10.1186/1471-2105-12-323

Li, X., Kang, Y. Q., Luo, Y. L., Zhang, K. Q., Zou, C. G., and Liang, L. M. (2017). The NADPH Oxidase AoNoxA in Arthrobotrys Oligospora Functions as an Initial Factor in the Infection of Caenorhabditis Elegans. J. Microbiol. 55, 885891. doi: 10.1007/s12275-017-7169-X

Liu, T., Huang, Y., Chen, X. X., Long, X., Yang, Y. H., Zhu, M. L., et al. (2020). Comparative Transcriptomics Reveals Features and Possible Mechanisms of Glucose-Mediated Soil Fungistasis Relief in Arthrobotrys Oligospora. Front. Microbiol. 10, 3143. doi: 10.3389/fmicb. 2019.03143

Liu, X. H., Lu, J. P., Zhang, L., Dong, B., Min, H., and Lin, F. C. (2007). Involvement of a Magnaporthe Grisea Serine/Threonine Kinase Gene, MgATG1, in Appressorium Turgor and Pathogenesis. Eukaryot. Cell 6, $997-$ 1005. doi: 10.1128/EC.00011-07

Livak, K. J., and Schmittgen, T. D. (2001). Analysis of Relative Gene Expression Data Using Real-Time Quantitative PCR and the $2^{-\Delta \Delta C T}$ Method. Methods 25, 402-408. doi: 10.1006/meth.2001.1262

Long, X., He, N. M., Tan, L. X., Yang, Y. H., Zhou, J. P., Liu, Z. Y., et al. (2021). Methylglyoxal has Different Impacts on the Fungistatic Roles of Ammonia and Benzaldehyde, and Lactoylglutathione Lyase is Necessary for the Resistance of Arthrobotrys Oligospora to Soil Fungistasis. Front. Cell Infect. Microbiol. 11, 640823. doi: $10.3389 /$ fcimb.2021.640823

Lu, J. P., Liu, X. H., Feng, X. X., Min, H., and Lin, F. C. (2009). An Autophagy Gene, MgATG5, is Required for Cell Differentiation and Pathogenesis in Magnaporthe Oryzae. Curr. Genet. 55, 461-473. doi: 10.1007/s00294-0090259-5

Lv, W. Y., Wang, C. Y., Yang, N., Que, Y. W., Talbot, N. J., and Wang, Z. Y. (2017). Genome-Wide Functional Analysis Reveals That Autophagy is Necessary for Growth, Sporulation, Deoxynivalenol Production and Virulence in Fusarium Graminearum. Sci. Rep. 7, 11062. doi: 10.1038/s41598-017-11640-Z

Ma, Y., Yang, X., Xie, M., Zhang, G., Yang, L., Bai, N., et al. (2020). The Arf-GAP AoGlo3 Regulates Conidiation, Endocytosis, and Pathogenicity in the Nematode-Trapping Fungus Arthrobotrys Oligospora. Fungal Genet. Biol. 138, 103352. doi: 10.1016/j.fgb.2020.103352

Ma, N., Zhao, Y., Wang, Y., Yang, L., Li, D., Yang, J., et al. (2021). Functional Analysis of Seven Regulators of G Protein Signaling (RGSs) in the NematodeTrapping Fungus Arthrobotrys Oligospora. Virulence 12, 1825-1840. doi: 10.1080/21505594.2021.1948667

Memisoglu, G., Eapen, V. V., Yang, Y., Klionsky, D. J., and Haber, J. E. (2019). PP2C Phosphatases Promote Autophagy by Dephosphorylation of the Atg1 Complex. Proc. Natl. Acad. Sci. U.S.A. 116, 1613-1620. doi: 10.1073/ pnas. 1817078116

Michán, S., Lledías, F., and Hansberg, W. (2003). Asexual Development is Increased in Neurospora Crassa Cat-3-Null Mutant Strains. Eukaryot. Cell 2, 798-808. doi: 10.1128/EC.2.4.798-808.2003 
Nadal, M., and Gold, S. E. (2010). The Autophagy Genes ATG8 and ATG1 Affect Morphogenesis and Pathogenicity in Ustilago Maydis. Mol. Plant Pathol. 11, 463-478. doi: 10.1111/j.1364-3703.2010.00620.x

Nakatogawa, H. (2020). Mechanisms Governing Autophagosome Biogenesis. Nat. Rev. Mol. Cell Biol. 21, 439-458. doi: 10.1038/s41580-020-0241-0

Nanji, T., Liu, X., Chew, L. H., Li, F. K., Biswas, M., Yu, Z. Q., et al. (2017). Conserved and Unique Features of the Fission Yeast Core Atg1 Complex. Autophagy 13, 2018-2027. doi: 10.1080/15548627.2017.1382782

Niu, X. M., and Zhang, K. Q. (2011). Arthrobotrys Oligospora: A Model Organism for Understanding the Interaction Between Fungi and Nematodes. Mycology 2, 59-78. doi: 10.1080/21501203.2011.562559

Nordbring-Hertz, B. (2004). Morphogenesis in the Nematode-Trapping Fungus Arthrobotrys Oligospora - an Extensive Plasticity of Infection Structures. Mycologist 18, 125-133. doi: 10.1017/S0269915X04003052

Nordbring-Hertz, B., Jansson, H. B., and Tunlid, A. (2006). "Nematophagous Fungi," in In Encyclopedia of Life Sciences (Chichester: John Wiley \& Sons, Ltd).

Park, G., Colot, H. V., Collopy, P. D., Krystofova, S., Crew, C., Ringelberg, C., et al. (2011). High-Throughput Production of Gene Replacement Mutants in Neurospora Crassa. Methods Mol. Biol. 722, 179-189. doi: 10.1007/978-161779-040-9_13

Pollack, J. K., Harris, S. D., and Marten, M. R. (2009). Autophagy in Filamentous Fungi. Fungal Genet. Biol. 46, 1-8. doi: 10.1016/j.fgb.2008.10.010

Ren, W., Zhang, Z., Shao, W., Yang, Y., Zhou, M., and Chen, C. (2017). The Autophagy-Related Gene BcATG1 is Involved in Fungal Development and Pathogenesis in Botrytis Cinerea. Mol. Plant Pathol. 18, 238-248. doi: 10.1111/ mpp. 12396

Stephan, J. S., Yeh, Y. Y., Ramachandran, V., Deminoff, S. J., and Herman, P. K. (2009). The Tor and PKA Signaling Pathways Independently Target the Atg1/ Atg13 Protein Kinase Complex to Control Autophagy. Proc. Natl. Acad. Sci. U.S.A. 106, 17049-17054. doi: 10.1073/pnas.0903316106

Stjepanovic, G., Davies, C. W., Stanley, R. E., Ragusa, M. J., Kim, D. J., and Hurley, J. H. (2014). Assembly and Dynamics of the Autophagy-Initiating Atgl Complex. Proc. Natl. Acad. Sci. U.S.A. 111, 12793-12798. doi: 10.1073/ pnas.1407214111

Sun, X., Wang, F., Lan, N., Liu, B., Hu, C., Xue, W., et al. (2019). The Zn(II)2Cys6Type Transcription Factor ADA-6 Regulates Conidiation, Sexual Development, and Oxidative Stress Response in. Neurospora crassa. Front. Microbiol. 10, 750. doi: 10.3389/fmicb.2019.00750

Su, H., Zhao, Y., Zhou, J., Feng, H., Jiang, D., Zhang, K. Q., et al. (2017). Trapping Devices of Nematode-Trapping Fungi: Formation, Evolution, and Genomic Perspectives. Biol. Rev. 92, 357-368. doi: 10.1111/brv.12233

Talbot, N. J., Ebbole, D. J., and Hamer, J. E. (1993). Identification and Characterization of Mpg1, a Gene Involved in Pathogenicity From the Rice Blast Fungus Magnaporthe Grisea. Plant Cell 5, 1575-1590. doi: 10.1105/ tpc.5.11.1575

Tunlid, A., Ahman, J., and Oliver, R. P. (1999). Transformation of the Nematode Trapping Fungus Arthrobotrys Oligospora. FEMS Microbiol. Lett. 173, 111116. doi: 10.1111/j.1574-6968.1999.tb13491.x

Wang, N., Yoshida, Y., and Hasunuma, K. (2007). Loss of Catalase-1 (Cat-1) Results in Decreased Conidial Viability Enhanced by Exposure to Light in Neurospora Crassa. Mol. Genet. Genomics 277, 13-22. doi: 10.1007/s00438006-0170-4

Wei, L. X., Zhang, H. X., Tan, J. L., Chu, Y. S., Li, N., Xue, H. X., et al. (2011). Arthrobotrisins A-C, Oligosporons From the Nematode-Trapping Fungus Arthrobotrys Oligospora. J. Nat. Prod. 74, 1526-1530. doi: 10.1021/np200187z

Xie, M. H., Bai, N., Yang, J. L., Jiang, K. X., Zhou, D. X., Zhao, Y. N., et al. (2020). Protein Kinase Ime2 is Required for Mycelial Growth, Conidiation, Osmoregulation, and Pathogenicity in Nematode-Trapping Fungus Arthrobotrys Oligospora. Front. Microbiol. 10, 3065. doi: 10.3389/ fmicb.2019.03065

Xie, M., Wang, Y., Tang, L., Yang, L., Zhou, D., Li, Q., et al. (2019). AoStuA, an APSES Transcription Factor, Regulates the Conidiation, Trap Formation, Stress Resistance and Pathogenicity of the Nematode-Trapping Fungus Arthrobotrys oligospora. Environ. Microbiol. 21, 4648-4661. doi: 10.1111/ $1462-2920.14785$

Xie, M., Yang, J., Jiang, K., Bai, N., Zhu, M., Zhu, Y., et al. (2021). AoBckl and AoMkk1 are Necessary to Maintain Cell Wall Integrity, Vegetative Growth,
Conidiation, Stress Resistance, and Pathogenicity in the Nematode-Trapping Fungus Arthrobotrys Oligospora. Front. Microbiol. 12, 649582. doi: 10.3389/ fmicb.2021.649582

Yanagisawa, S., Kikuma, T., and Kitamoto, K. (2013). Functional Analysis of Aoatg1 and Detection of the Cvt Pathway in Aspergillus Oryzae. FEMS Microbiol. Lett. 338, 168-176. doi: 10.1111/1574-6968.12047

Yang, L., Li, X., Xie, M., Bai, N., Yang, J., Jiang, K., et al. (2021). Pleiotropic Roles of Ras GTPases in the Nematode-Trapping Fungus Arthrobotrys Oligospora Identified Through Multi-Omics Analyses. iScience 24, 102820. doi: 10.1016/ j.isci.2021.102820

Yang, X., Ma, N., Yang, L., Zheng, Y., Zhen, Z., Li, Q., et al. (2018). Two Rab GTPases Play Different Roles in Conidiation, Trap Formation, Stress Resistance, and Virulence in the Nematode-Trapping Fungus Arthrobotrys Oligospora. Appl. Microbiol. Biotechnol. 102, 4601-4613. doi: 10.1007/s00253018-8929-1

Yang, C. T., Vidal-Diez de Ulzurrun, G., Gonçalves, A. P., Lin, H. C., Chang, C. W., Huang, T. Y., et al. (2020). Natural Diversity in the Predatory Behavior Facilitates the Establishment of a Robust Model Strain for Nematode-Trapping Fungi. Proc. Natl. Acad. Sci. U.S.A. 117, 6762-6770. doi: 10.1073/ pnas. 1919726117

Yang, J., Wang, L., Ji, X., Feng, Y., Li, X., Zou, C., et al. (2011). Genomic and Proteomic Analyses of the Fungus Arthrobotrys Oligospora Provide Insights Into Nematode-Trap Formation. PloS Pathog. 7, e1002179. doi: 10.1371/ journal.ppat.1002179

Yang, J. K., Yu, Y., Li, J., Zhu, W., Geng, Z. Y., Jiang, D. W., et al. (2013). Characterization and Functional Analyses of the Chitinase-Encoding Genes in the Nematode-Trapping Fungus Arthrobotrys Oligospora. Arch. Microbiol. 195, 453-462. doi: 10.1007/s00203-013-0894-6

Ying, S. H., and Feng, M. G. (2019). Insight Into Vital Role of Autophagy in Sustaining Biological Control Potential of Fungal Pathogens Against Pest Insects and Nematodes. Virulence 10, 429-437. doi: 10.1080/21505594.2018.1518089

Ying, S. H., Liu, J., Chu, X. L., Xie, X. Q., and Feng, M. G. (2016). The AutophagyRelated Genes BbATG1 and BbATG8 Have Different Functions in Differentiation, Stress Resistance and Virulence of Mycopathogen Beauveria Bassiana. Sci. Rep. 6, 26376. doi: 10.1038/srep26376

Yu, X., Hu, X., Pop, M., Wernet, N., Kirschhöfer, F., Brenner-Weiß, G., et al. (2021). Fatal Attraction of Caenorhabditis elegans to predatory fungi through 6-methyl-salicylic acid. Nat. Commun. 12, 5462. doi: 10.1038/s41467-02125535-1

Zhang, W., Chen, J., Fan, Y., Hussain, M., Liu, X., and Xiang, M. (2021). The E3Ligase AoUBR1 in N-End Rule Pathway is Involved in the Vegetative Growth, Secretome, and Trap Formation in Arthrobotrys Oligospora. Fungal Biol. 125, 532-540. doi: 10.1016/j.funbio.2021.02.003

Zhang, G., Zheng, Y., Ma, Y., Yang, L., Xie, M., Zhou, D., et al. (2019). The Velvet Proteins VosA and VelB Play Different Roles in Conidiation, Trap Formation, and Pathogenicity in the Nematode-Trapping Fungus Arthrobotrys oligospora. Front. Microbiol. 10, 1917. doi: 10.3389/fmicb.2019.01917

Zhao, X., Wang, Y., Zhao, Y., Huang, Y., Zhang, K. Q., and Yang, J. (2014). Malate Synthase Gene AoMls in the Nematode-Trapping Fungus Arthrobotrys Oligospora Contributes to Conidiation, Trap Formation, and Pathogenicity. Appl. Microbiol. Biotechnol. 98, 2555-2563. doi: 10.1007/s00253-013-5432-6

Zhen, Z., Xing, X., Xie, M., Yang, L., Yang, X., Zheng, Y., et al. (2018). MAP Kinase Slt2 Orthologs Play Similar Roles in Conidiation, Trapformation, and Pathogenicity in Two Nematode-Trapping Fungi. Fungal Genet. Biol. 116, 42-50. doi: 10.1016/j.fgb.2018.04.011

Zhou, D. X., Xie, M., Bai, N., Yang, L., Zhang, K. Q., and Yang, J. (2020). The Autophagy-Related Gene Aoatg4 Regulates Hyphal Growth, Sporulation, Autophagosome Formation, and Pathogenicity in Arthrobotrys Oligospora. Front. Microbiol. 11, 592524. doi: 10.3389/fmicb.2020.592524

Zhou, D., Zhu, Y., Bai, N., Yang, L., Xie, M., Yang, J., et al. (2021). AoATG5 Plays Pleiotropic Roles in Vegetative Growth, Cell Nucleus Development, Conidiation, and Virulence in the Nematode-Trapping Fungus Arthrobotrys Oligospora. Sci. China Life Sci. doi: 10.1007/s11427-020-1913-9

Zhu, X. M., Li, L., Wu, M., Liang, S., Shi, H. B., Liu, X. H., et al. (2019). Current Opinions on Autophagy in Pathogenicity of Fungi. Virulence 10, 481-489. doi: 10.1080/21505594.2018.1551011

Zhu, J., Yu, X., Xie, B., Gu, X., Zhang, Z., and Li, S. (2013). Transcriptomic Profiling-Based Mutant Screen Reveals Three New Transcription Factors 
Mediating Menadione Resistance in Neurospora Crassa. Fungal Biol. 117, 422430. doi: 10.1016/j.funbio.2013.04.006

Conflict of Interest: The authors declare that the research was conducted in the absence of any commercial or financial relationships that could be construed as a potential conflict of interest.

Publisher's Note: All claims expressed in this article are solely those of the authors and do not necessarily represent those of their affiliated organizations, or those of the publisher, the editors and the reviewers. Any product that may be evaluated in this article, or claim that may be made by its manufacturer, is not guaranteed or endorsed by the publisher.

Copyright (c) 2022 Zhou, Zhu, Bai, Xie, Zhang and Yang. This is an open-access article distributed under the terms of the Creative Commons Attribution License (CC BY). The use, distribution or reproduction in other forums is permitted, provided the original author(s) and the copyright owner(s) are credited and that the original publication in this journal is cited, in accordance with accepted academic practice. No use, distribution or reproduction is permitted which does not comply with these terms. 